\title{
ROLE OF STEREOPSIS LOSSES IN OLDER ADULTS' PERFORMANCE ON THE FINE-GRAIN MOVEMENT ILLUSION TASK
}

\author{
by \\ Marlena Pearson \\ Honours Bachelor of Science, Psychology, Nipissing University, North Bay, Ontario, 2014 \\ Honours Bachelor of Arts, Criminal Justice, Nipissing University, North Bay, Ontario, 2014
}

\author{
A thesis \\ presented to Ryerson University \\ in partial fulfillment of the \\ requirement for the degree of \\ Master of Arts \\ in the program of \\ Psychology
}

Toronto, Ontario, Canada, 2017

(C) Marlena Pearson, 2017 


\section{Author's Declaration \\ AUTHOR'S DECLARATION FOR ELECTRONIC SUBMISSION OF A THESIS}

I hereby declare that I am the sole author of this thesis. This is a true copy of the thesis, including any required final revisions, as accepted by my examiners.

I authorize Ryerson University to lend this thesis to other institutions or individuals for the purpose of scholarly research

I further authorize Ryerson University to reproduce this thesis by photocopying or by other means, in total or in part, at the request of other institutions or individuals for the purpose of scholarly research.

I understand that my thesis may be made electronically available to the public. 


\title{
Role of Stereopsis Losses in Older Adults' Performance \\ on the Fine-Grain Movement Illusion Task
}

Master of Arts, 2017

\author{
Marlena Pearson \\ Psychology, Ryerson University
}

\begin{abstract}
Accurate motion perception is necessary for older adults to safely navigate their environments. Yet it is not clear how stereopsis losses contribute to findings of motion perception deficits in older adults. To assess the contribution of stereopsis losses, three groups (younger adults, older adults with intact stereopsis, older adults with poor stereopsis) were recruited for a fine-grain movement task. The distance participants perceived a dot to move across a computer screen was assessed using a staircase procedure. While all participants perceived the dot to move further than the actual distance, older adults with poor stereopsis showed more exaggeration in their estimates than younger adults and older adults with intact stereopsis. However, the groups did not differ in the intraindividual variability of their estimates. These results suggest stereopsis losses in the context of aging may signal neural or oculomotor changes that result in reduced accuracy of positional perception.
\end{abstract}




\section{Acknowledgments}

The completion of my MA thesis was made possible through the extraordinary support provided at Ryerson University. First and foremost, I greatly appreciate the guidance of Dr. Maureen Reed, my thesis advisor. Maureen's high standards and attention to detail were instrumental in my success. Many thanks to Dr. Todd Girard and Dr. Lixia Yang for agreeing to serve on my thesis defense committee. During the process of refining my thesis, their feedback was invaluable. I would also like to thank Carson Pun for his technical assistance with programming in E-Prime. Finally, I gratefully acknowledge the support of my partner (Alain Carlson), friends, and family. I am excited to begin my $\mathrm{PhD}$ work this fall! 


\section{Table of Contents}

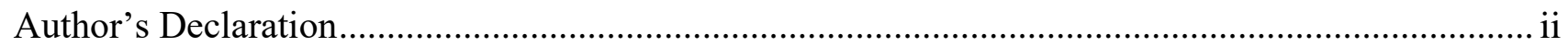

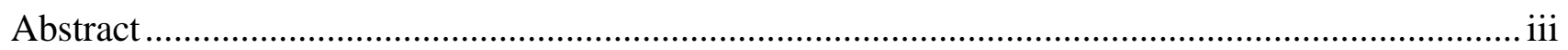

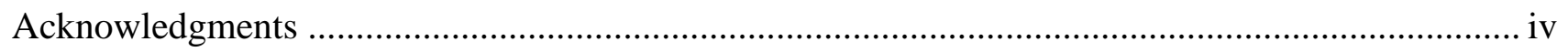

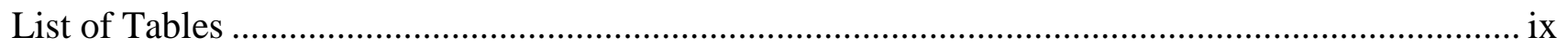

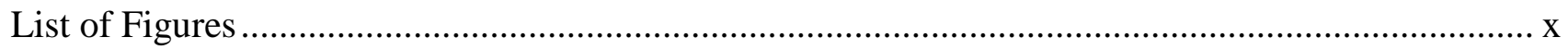

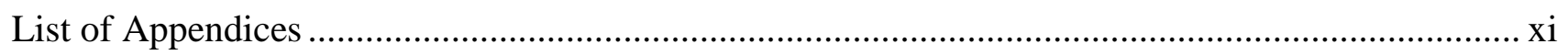

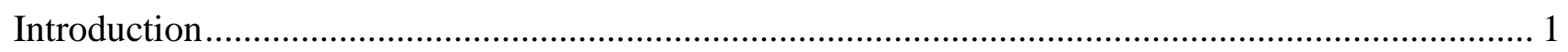

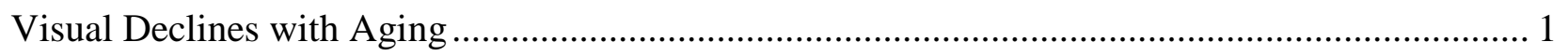

Effects of Aging on Structure of the Retina and Visual Cortex ................................................ 2

Neural Effects of Aging: Neural Noise Hypothesis........................................................... 4

Age-Related Neural Changes Affecting Motion Perception ................................................. 6

Mechanisms by Which Stereopsis Losses May Indicate Losses in Motion Perception ................. 7

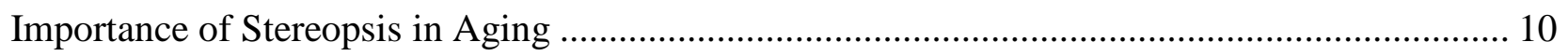

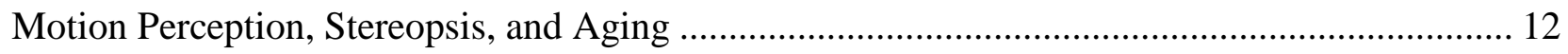

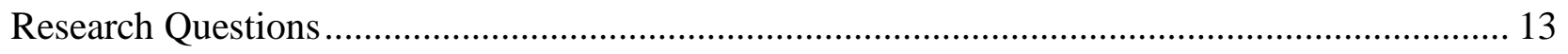

Research Question 1: Do older adults show less accuracy in their motion estimates than young adults, and are these differences influenced by older adults' stereoacuity? ....................... 13

Research Question 2: Do older adults show less precision in their motion estimates than young adults, and are these differences influenced by older adults' stereoacuity? ...................... 13 
Research Question 3: Is the difference in the magnitude of change in motion estimates between central and peripheral vision different in younger and older adults, and are these differences influenced by older adults' stereoacuity? ............................................................................. 14

Research Question 4: For older adults, are changes in age and stereoacuity associated with changes in central and peripheral performance?.............................................................. 15

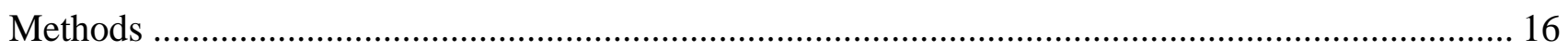

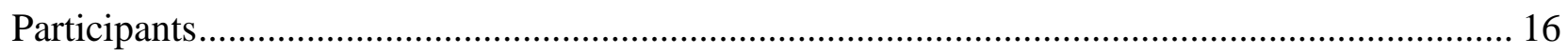

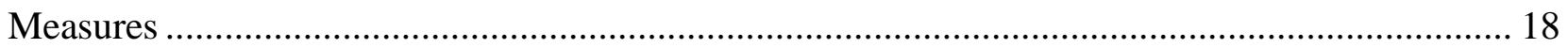

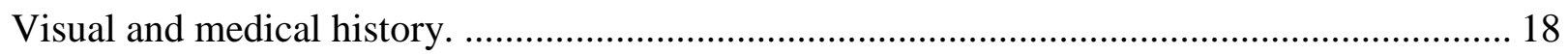

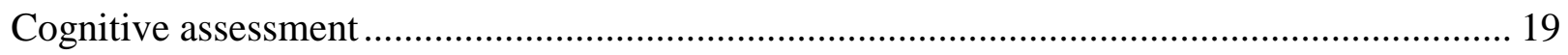

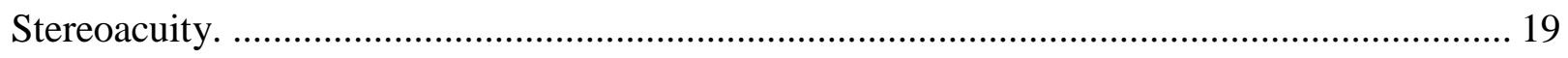

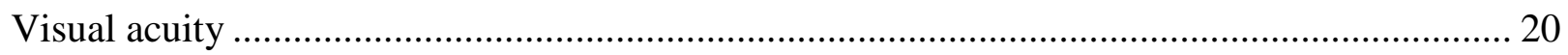

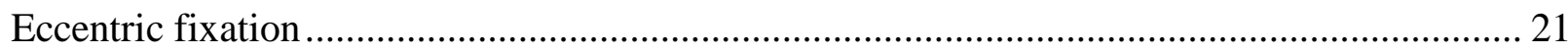

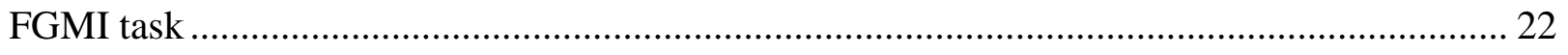

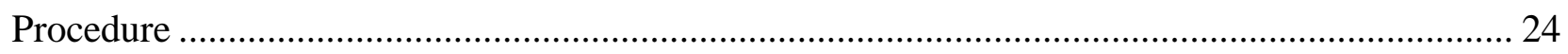

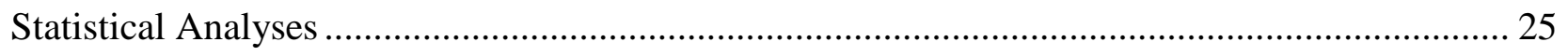

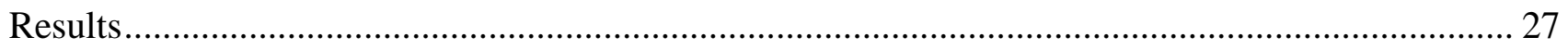

Research Question 1: Do older adults show less accuracy in their motion estimates than young adults, and are these differences influenced by older adults' stereoacuity? 
Research Question 2: Do older adults show less precision in the linear extent of their movement estimates than young adults, and are these differences influenced by older adults' stereoacuity?

Research Question 3: Is the difference in the magnitude of change in motion estimates between central and peripheral vision different in younger and older adults, and are these differences influenced by older adults' stereoacuity?

Research Question 4: For older adults, are changes in age and stereoacuity associated with changes in central and peripheral performance? 36

Discussion 38

Relationships between Aging Alone and Central and Peripheral Accuracy and Precision of Motion Estimates 38

Relationships between Stereoacuity Losses in the Context of Aging and Central and Peripheral Accuracy and Precision of Motion Estimates. 44

Limitations 50

Future Directions 53

Significance 54

Appendices 56

Appendix A: Visual and Medical History (Older Adults) ............................................................. 56

Appendix B: Visual and Medical History (Younger Adults) ........................................................ 58

Appendix C: Montreal Cognitive Assessment (MoCA) ................................................................ 59

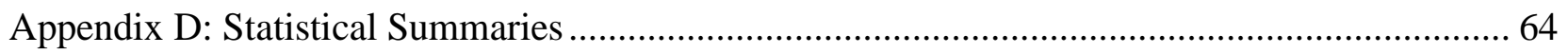




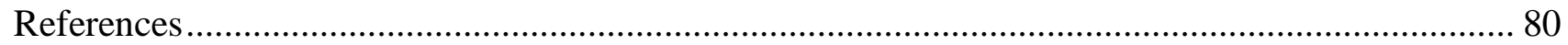

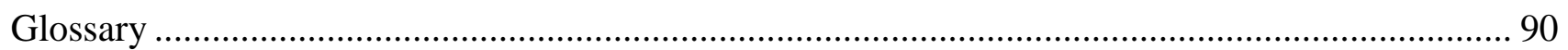




\section{List of Tables}

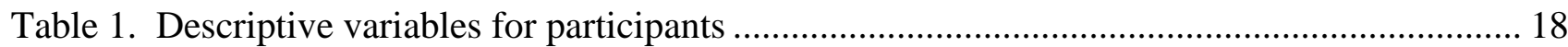




\section{List of Figures}

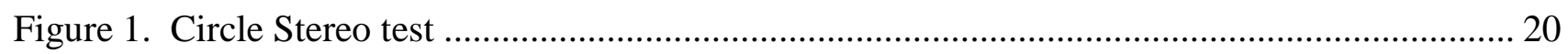

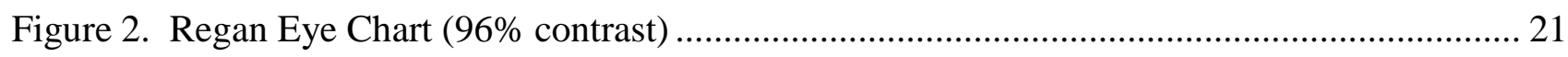

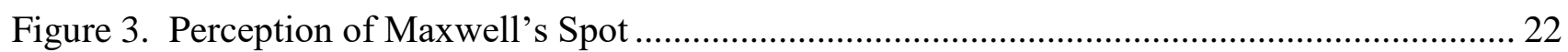

Figure 4. Sample presentation of the dots and a standard line at the beginning of each trial for the

FGMI task

Figure 5. Median central vision accuracy for the younger adults, older adults with intact stereopsis, and older adults with poor stereopsis

Figure 6. Median peripheral accuracy for the younger adults, older adults with intact stereopsis, and older adults with poor stereopsis.

Figure 7. Median central vision precision for the younger adults, older adults with intact stereopsis, and older adults with poor stereopsis

Figure 8. Median peripheral vision precision for the younger adults, older adults with intact stereopsis, and older adults with poor stereopsis

Figure 9. Median vision accuracy difference scores (peripheral vision accuracy - central vision accuracy) for the younger adults, older adults with intact stereopsis, and older adults with poor stereopsis

Figure 10. Median vision precision difference scores (peripheral vision precision - central vision precision) for the younger adults, older adults with intact stereopsis, and older adults with poor stereopsis 


\section{List of Appendices}

Appendix A: Visual and Medical History (Older Adults) ......................................................... 56

Appendix B: Visual and Medical History (Younger Adults) ................................................... 58

Appendix C: Montreal Cognitive Assessment (MoCA) ........................................................ 59

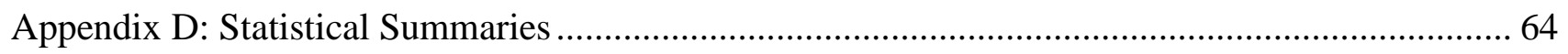




\section{Role of Stereopsis Losses in Older Adults' Performance \\ on the Fine-Grain Movement Illusion Task}

Accurate motion perception is necessary for older adults to safely navigate their

environments. While some researchers have found motion perception is negatively affected by aging (Spear, 1993), others have not (Faubert, 2002). Faubert (2002) suggested these contrasting findings could be related to differences in stimulus complexity, since older adults may have processing deficits for complex stimuli. Yet some researchers have found that differences in stereoacuity $^{1}$ are associated with poor performance on some motion perception tasks and argue that motion perception and stereopsis may share neural mechanisms (Reed \& Burdett, 2002; Thompson \& Nawrot, 1999). Since aging is associated with losses in stereoacuity (Lee \& Koo, 2005), it is possible that losses in stereoacuity signal changes in neuron populations/mechanisms involved in motion processing, which could account for some findings of motion perception deficits in older adults. The present research addressed this possibility by using the fine-grain movement illusion (FGMI) task to determine if it is only older adults with stereoacuity losses that show motion perception losses. Specifically, accuracy and precision of motion estimates in central vision and peripheral vision were compared between younger adults, older adults with intact stereopsis, and older adults with poor stereopsis. Across the older adults, it was also explored whether changes in age and stereoacuity were associated with changes in central and peripheral motion performance.

\section{Visual Declines with Aging}

Aging is associated with the progressive development of physiological impairments across a range of domains (López-Otín, Blasco, Partridge, Serrano, \& Kroemer, 2013; Niccoli \&

\footnotetext{
${ }^{1}$ Definitions for bolded terms are provided in the glossary. Only the first occurrence of each term is bolded.
} 
Partridge, 2012). However, the extent to which individuals experience these age-related changes depends on a range of genetic and environmental factors (Brooks-Wilson, 2013; Lin, Epel, \& Blackburn, 2012). On a cognitive level, there are declines in processing speed, reasoning, memory, executive function, and attention (Deary et al., 2009). According to the inhibitory deficit hypothesis (Hasher \& Zacks, 1988), older adults have more difficulty inhibiting irrelevant information during task performance, which could impact the performance of everyday activities. These decrements in cognitive function and attention could negatively affect visual abilities, especially for complex stimuli (Faubert, 2002; Owsley, 2011).

Some of the sensory and perceptual declines in vision that occur with aging are the result of disease processes such as glaucoma, age-related macular degeneration, and diabetic retinopathy. However, most declines in vision are a normal part of the aging process (Owsley, 2011). In terms of low-level structural changes that occur with normal aging, the curvature of the cornea changes from "with the rule" astigmatism (i.e., the vertical meridian is steeper than the horizontal meridian) to "against the rule" astigmatism (i.e., the horizontal meridian is steeper than the vertical meridian), which makes it more difficult to resolve vertically oriented targets (Salvi, Akhtar, \& Currie, 2006). Also, the lens loses transparency and its ability to accommodate light (Michael \& Bron, 2011). Older adults show reduced visual acuity, decreased colour vision, and impaired processes for dark and light adaptation (Spear, 1993). As well, useful field of view, the area from which visual information can be extracted without moving the head or eyes, has been shown to decline with aging (Sekuler, Bennett, \& Mamelak, 2000).

\section{Effects of Aging on Structure of the Retina and Visual Cortex}

Aging is also associated with changes in the structure of the retina and visual cortex. In both younger and older adults, the number of cones decreases with increasing eccentricity from the 
fovea (Bonnel, Mohand-Said, \& Sahel, 2003; Lombardo, Serrao, Ducoli, \& Lombardo, 2013). However, Gao \& Hollyfield (1992), in a sample whose age range (18-95 years) contained the age range of the younger adults (18-25 years) and older adults (66-89 years) in the present study, found aging was associated with significant decreases in the number of equatorial cones, equatorial rods, retinal pigment epithelial cells, and cells in the ganglion layer. Overall, photoreceptor loss was less in the fovea than in the periphery, with no significant decreases in the density of cone and retinal pigment epithelial cells in the foveal centre. In contrast, Kim, Tom, and Spear (1996) found no total or regional differences in the density, number, and soma sizes of retinal ganglion cells between younger and older rhesus monkeys. These contrasting findings may be related to species differences. To further examine the distribution of cones in the peripheral retina, Kimble and Williams (2000) measured the density and arrangement of cones in the nasal mid-periphery and temporal mid-periphery using a sample of 24 human donors between the ages of 15 and 83 . They found a small decline with aging in the regularity of the cone mosaic, but it was limited to the nasal mid-periphery.

Changes in the visual cortex with aging may also affect cortical magnification factor $(M) . M$ refers to the number of millimetres of visual cortex surface area that process stimuli per degree of visual angle (Cowey \& Rolls, 1974). $M$ decreases linearly with eccentricity; put simply, fewer and fewer visual cortex neurons are available to process visual stimuli from a given area of the visual field as one moves towards the periphery (Strasburger, Rentschler, \& Jüttner, 2011). Thus, the size of the receptive field for each visual cortex neuron increases with eccentricity. In a study examining the effects of healthy aging on primary visual cortex, Brewer and Barton (2012) used fMRI data to model population receptive field changes in the brain. For areas of V1 related to the processing of foveal vision from 0 to 3 degrees eccentric from fixation, aging was 
associated with decreases in surface area and increases in neuronal receptive field sizes. The authors also found that older participants had decreased V1 coherence from 7 to 10 degrees eccentric from fixation (i.e., the neuronal responses in V1 for older adults were less correlated with the modeled neuronal responses than were the neuronal responses in V1 for younger adults), which they attributed to greater losses of peripheral photoreceptors than central photoreceptors with aging.

The structural integrity of myelin in the primary visual cortex may also become compromised with aging. In a study comparing myelin sheaths of the primary visual cortex in younger (5-10 years old) and older (25-33 years old) rhesus monkeys, aging was associated with changes in the structure of myelin, such as bulging and ballooning (Peters, Moss, \& Sethares, 2000). These changes in myelin were significantly correlated with poorer performance on three visual recognition memory tasks. The thickness of myelin sheaths in the primary visual cortex has also been found to increase with aging in rhesus monkeys (Peters et al., 2000; Peters, Sethares, \& Killiany, 2001), which could reflect dysfunctional changes with aging that make the sheath too large for the axon it surrounds (Peters et al., 2000) or could reflect compensatory processes intended to offset sheath degeneration or to promote remyelination (Peter et al., 2001). However, a contrasting review, citing evidence of no significant changes in myelination or other structural properties of the visual cortex with aging, concluded changes in neuron function and communication (e.g., decreases in levels of neurotransmitters) are primarily responsible for agerelated declines in the perception of contrast and motion (Andersen, 2012).

\section{Neural Effects of Aging: Neural Noise Hypothesis}

A significant body of research suggests aging is associated with higher intraindividual variability in task performance and neural activity (see MacDonald, Nyberg, \& Bäckman, 2006). 
According to the neural noise hypothesis, neural noise increases with aging (Gregory, 1957), which may contribute to these increases in intraindividual variability. However, the neural noise hypothesis is not the only plausible explanation for higher intraindividual variability with aging. Salthouse and Lichty (1985), after failing to find evidence supporting the neural noise hypothesis in two of the three dependent measures, suggested that the older adults may have used strategies to compensate for age-related deficits in information processing. Shifts in these strategies during tasks could also explain findings of greater intraindividual variability in older adults' performance. Further, some researchers argue that increases in intraindividual variability for neural measures could be the manifestation of adaptive mechanisms intended to maintain neural performance with aging, since neural connections formed in the presence of noise are more resistant to disruptions (Garrett, Kovacevic, McIntosh, \& Grady, 2010; Garrett et al., 2013). By this account, increasing neural noise with aging could have protective effects on functioning. Voytek and colleagues (2015) attempted to address these alternate explanations for intraindividual variability by assessing $1 / f$ noise, a signal processing definition of noise based on the shape of the distribution of the frequencies that compose the signal. Eleven younger adults (20-30 years old) and 13 older adults (60-70 years old) were compared on their $1 / f$ noise using EEG and on their performance on a visual working memory task. The authors found aging was associated with more $1 / f$ noise, and that impairments in visual working memory performance were mediated by visual cortical $1 / f$ noise. These results suggest that aging may be associated with increases in neural noise that are not related to shifting cognitive strategies or adaptive neural mechanisms, and that predict poorer performance on visual working memory. 


\section{Age-Related Neural Changes Affecting Motion Perception}

Motion perception requires the coordinated action of several sensory and neural structures. An object in the environment reflects any light that it does not absorb. The cornea and lens then focus the light onto the retina at the back of the eye, which stimulates photoreceptors in the retina. The photoreceptors convert the light into impulses, and the optic nerve sends these impulses through the optic nerve and lateral geniculate nucleus of the thalamus to neurons in primary visual cortex. As the object moves across the visual field, photoreceptors and neuronal cells are sequentially stimulated, giving rise to the perception of a single object moving across the retina.

The magnocellular pathway deals with the "where" properties of this visual information (Livingstone \& Hubel, 1988). Motion perception, a primary function of the magnocellular pathway, involves the inference of speed and direction of visual elements in terms of first-order and second-order motion. The perception of first-order motion is related to changes in luminance whereas second-order motion is related to changes in qualities other than luminance, such as contrast or texture (Vaina \& Soloviev, 2004). The perception of motion that occurs when a pair of lights flash on-and-off in sequence, a classic demonstration of beta movement, is an example of first-order motion (Cavanagh \& Mather, 1989). Motion perception sensitivity in aging is impaired for both first-order and second-order motion processes, particularly in foveal vision (Faubert, 2002). Visual area middle temporal (MT)/V5, a major cortical area of the magnocellular pathway, undergoes significant changes with aging. Relative to young macaques, the area MT neurons of old macaques are less selective to direction (Liang et al., 2010) and differences in speed (Yang, Zhang, et al., 2009). Neurons of old macaques in area MT and the primary visual cortex (V1) are also less sensitive to contrast (Yang et al., 2008), and have higher 
response variability (Yang, Liang, Li, Wang, \& Zhou, 2009). Older macaque monkeys have also shown increased neuronal response latency in the inferior temporal cortex, a structure involved in object and shape recognition (Csete, Bognár, Csibri, Kaposvári, \& Sáry, 2015). Chang et al. (2015) found significant reductions in the size of V1, V2, and $\mathbf{V 3}$ of the visual cortex in older adults relative to younger adults. The correlation between older adults' performance on the texture discrimination task and the size of $\mathrm{V} 3$ suggests negative functional consequences of V3 atrophy for motion perception, as spatiotemporal changes in texture can be used to perceive motion (Chubb \& Sperling, 1989). Also, V1, V2, and V3 are functionally connected to area MT (Yeo et al., 2011).

\section{Mechanisms by Which Stereopsis Losses May Indicate Losses in Motion Perception}

Some researchers have suggested that stereopsis and motion perception share a neural mechanism responsible for positional information (Reed \& Burdett, 2002). fMRI studies have provided some insights into the neuron populations that support the processing of this positional information. The findings of Claeys, Lindsey, De Schutter, and Orban (2003) suggest the existence of two motion-processing systems: (1) a contralateral region spanning from area MT to the dorsal intraparietal sulcus and the superior temporal sulcus that is involved in processing first-order and second-order motion, and (2) a bilateral region in the inferior parietal lobule involved in the tracking of higher-level salient features. Within that first motion-processing system, there is also evidence for separate neural mechanisms for first-order and second-order motion perception, as there are separate populations of neurons within area MT that respond to first-order and second-order motion (Ashida, Lingnau, Wall, \& Smith, 2007). Also, sensitivity to depth has been found in all areas of the primary visual cortex (Neri, Bridge, \& Heeger, 2004), and one study of macaques found 93\% of area MT cells respond to disparity (DeAngelis \& Uka, 
2003). Thus, although this idea has not been tested in a study contrasting neural measures of older adults with intact stereopsis and older adults with poor stereopsis, it is possible that stereopsis losses may signal structural or functional deficits in the neuron populations that support motion processing, which could account for some findings of motion perception deficits with aging and the relationship between stereoacuity losses and FGMI exaggeration.

Stereopsis losses could also signal changes in oculomotor function. A retrospective study of 500 older optometry patients found disorders of binocular vision or eye movement (e.g., strabismus), which are often associated with or defined by deficits in oculomotor function, in about half of the older adults over the age of 60 (Leat et al., 2013). In a sample of 12 older adults with age-related macular degeneration and 16 younger adults with normal vision, TaritaNistor, Brent, Steinbach, and González (2012) used eye tracking to compare steadiness of fixation on a target during binocular and monocular viewing. The younger adults showed no significant horizontal or vertical drift in fixation in the viewing eye(s) under both monocular and binocular conditions. Interestingly, the older adults showed no significant vertical drift in the viewing eye(s), and they only showed more horizontal drift than the younger adults in the viewing eye during monocular fixation with the eye with worse visual acuity. Although the older adults in this study had age-related macular degeneration, this finding could suggest that monocular viewing conditions expose subtle oculomotor deficits in older adults, deficits that are compensated for under binocular viewing conditions.

Another interesting finding by Tarita-Nistor and colleagues (2012) was the large proportion of the younger adults with normal vision and older adults with age-related macular degeneration that experienced horizontal phoria (eye drifting) in the covered eye under monocular viewing. When the right eye was covered, $63 \%$ of the younger adults showed exophoria (deviation of the 
eye outward) greater than 1 degree and 6\% showed esophoria (deviation of the eye inward) greater than 1 degree in that eye; when the left eye was covered, $69 \%$ of the younger adults showed exophoria greater than 1 degree and $13 \%$ showed esophoria greater than 1 degree in that eye. The older adults showed similar patterns of phoria when the better eye (the eye with better visual acuity) and the worse eye were covered: $67 \%$ of the older adults showed exophoria in the covered eye when the better or worse eye was covered, while $8 \%$ showed esophoria in the covered eye when the better eye was covered and $0 \%$ when the worse eye was covered. It is unclear whether such involuntary drifts in the covered eye affect visual tracking of an object under monocular conditions. However, voluntary eye movements are needed in the visual pursuit of moving objects to keep them focused on the fovea. Although there are no data to support these speculations, it is possible that involuntary drift in the covered eye could have represented meaningless eye movement resulting from fixation under unnatural monocular conditions. It is also possible that these involuntary movements could affect the distance an object is perceived to have moved by contributing perceptual noise that could interfere with estimating how far the viewing eye moved, particularly if the movement occurred over a relatively short distance. Finally, it is possible that these movements in the covered eye could be relatively consistent and thus have a relatively consistent effect on motion estimates, especially given that Tarita-Nistor and colleagues (2012) observed similar percentages of younger and older adults that showed more than 1 degree of exophoria in the covered eye.

While aging is associated with disorders of binocular vision and eye movement (Leat et al., 2013), some research suggests that older adults without these disorders can maintain fixation. An early study of 12 younger adults and 12 older adults with no visual pathologies, according to the results of thorough ophthalmological exams, found no significant differences in monocular 
fixation behaviour at the group level (Kosnik, Fikre, \& Sekuler, 1986). The authors attributed the observation of slightly larger variability along the horizontal meridian in the older adults than the younger adults, which did not reach statistical significance, to the performance of a few participants within the older adult group rather than to the effects of aging. It is possible that these individuals had stereopsis losses that were associated with this higher variability along the horizontal meridian, although there are no data to support this speculation since stereopsis measures were not provided. Another study by the same research group found both younger adults and older adults with no visual disorders could maintain fixation in an area much smaller than the fovea (Kosnik, Kline, Fikre, \& Sekuler, 1987), which also suggests aging alone may not affect fixation ability.

\section{Importance of Stereopsis in Aging}

As first described by Wheatstone (1838), stereopsis occurs because of the binocular disparity between our two forward facing eyes. In other words, the left and right eyes see slightly different images due to the physical distance between the eyes; the image on the retina of one eye is slightly disparate relative to the image on the retina of the other eye. The visual system's calculation of this disparity results in 3-dimensional perception. Although there are binocular depth neurons that are sensitive to a range of differences between these two images (Hubel \& Wiesel, 1970), it remains unclear specifically how the brain calculates the disparities between the left and right images (see Menz \& Freeman, 2003). There are two types of stereopsis described in the literature (Barry, 2012). Fine stereopsis is mainly based on static differences, and allows individuals to determine depth of objects within central vision during fine-motor tasks. Coarse stereopsis provides a sense of being immersed in one's surroundings, and is important for orientation in space while moving. 
Stereopsis may have been an important adaptation in evolutionary history. However, it is debated in the literature as to whether stereopsis is a vestigial ability with limited functional consequences in the modern world (Fielder \& Moseley, 1996). Binocular vision is not necessary to interpret depth; there are also monocular depth cues such as occlusion, relative size, and texture (Goldstein, 2014). For this reason, many individuals function quite well with one eye, or with little or no stereopsis. Even for complex tasks such as driving, both eyes are not required to hold a license. For example, Rubin et al. (2007), using crash data from state motor vehicle records, found stereoacuity was not a significant predictor of motor vehicle accidents in older adults.

However, aging is also associated with losses in stereoacuity. Lee and Koo (2005) measured stereoacuity in 80 participants without eye disease between the ages of 7 and 76 . Near stereoacuity was assessed using the TNO (The Netherlands Organization for Applied Scientific Research), Titmus, and Randot stereo tests while distance stereoacuity was assessed using the Mentor B-VAT II video acuity tester. Relative to the 7 to 10-year-old age group, the results of both the TNO stereo test and B-VAT II indicated significantly lower stereoacuity in participants between the ages of 51 and 76 while the Titmus and Randot results indicated lower stereoacuity but only in participants over the age of 70. Further, Wright and Wormald (1992) used the Frisby stereo test to assess stereoacuity in an elderly sample of 728 individuals over the age of 65, and they found that $27 \%$ had full stereopsis and $29 \%$ had no stereopsis. Finally, using a sample of observers between the ages of 18 and 83, Norman and colleagues (2008) found that older adults were unable to discriminate surface shapes in random dot stereograms with both high degrees of binocular disparity (51.5 minutes of arc) and large orientation differences between corresponding elements in the left and right images. 
Further, researchers have found poor stereoacuity is associated with fall-related outcomes in older adults. Impairments in stereopsis, contrast sensitivity, and low-contrast visual acuity are among the strongest risk factors for falls in older adults (Lord \& Dayhew, 2001). Older adults with poor stereoacuity are also at greater risk of recurrent falls (Nevitt, Cummings, Kidd, \& Black, 1989). Finally, poor stereoacuity is an important risk factor for hip fracture resulting from a fall in women (Cummings et al., 1995). These data suggest that losses in stereopsis may result in difficulties evaluating the environment, which could reflect underlying losses in positional mechanisms.

\section{Motion Perception, Stereopsis, and Aging}

Previous work with the FMGI suggests stereoacuity may affect motion perception. FGMI is a short-range motion illusion produced by sequentially flashing two dots separated by a few millimetres (Biederman-Thorson, Thorson, \& Lange, 1971). Typically, individuals report the perception of a dot travelling in the direction of the flash sequence that moves well beyond the spatial extent of the dots (i.e., FGMI exaggeration). The exaggerated perception is usually reported with 10-30 degrees peripheral viewing (Foster, Thorson, Mcllwain, \& BiedermanThorson, 1981); however, Reed and Burdett (2002) showed that the exaggeration can also be elicited in central vision in participants with strabismus (eye misalignment). In both cases, FGMI exaggeration has been attributed to cortical receptive field size and the distance between retinal cells. Yet, in participants with central retinal and cortical receptive field abnormalities, like those found in older adults, Reed and Burdett (2002) found these abnormalities did not entirely predict FGMI exaggeration. Instead, participants with larger losses in stereoacuity

showed more exaggerated FGMI perception. If, as Reed and Burdett (2002) and Thompson and Nawrot (1999) suggest, motion perception and stereopsis share neural mechanisms responsible 
for positional information, and, as Lee and Koo (2005) note, older adults often have losses in stereoacuity, then losses in motion perception with aging may be explained by stereoacuity losses.

\section{Research Questions}

To address the possibility that losses in motion perception with aging may be partially explained by stereoacuity losses, the present study compared accuracy and precision of FGMI perception in both central and peripheral vision for younger adults with intact stereopsis, older adults with intact stereopsis, and older adults with poor stereopsis. Four research questions were addressed by the present research.

\section{Research Question 1: Do older adults show less accuracy in their motion estimates than} young adults, and are these differences influenced by older adults' stereoacuity? Previous research has produced mixed results regarding whether motion perception is negatively affected by aging (Faubert, 2002; Spear, 1993). It is hypothesized that both younger adults and older adults with intact stereopsis will show significantly higher accuracy on motion estimates in central and peripheral vision than the older adults with poor stereopsis, since stereopsis losses may signal losses in positional mechanisms (Reed \& Burdett, 2002). It is unclear whether younger adults and older adults with intact stereopsis will significantly differ on accuracy. However, it is expected that the older adults with intact stereopsis will be more likely to show lower accuracy than the younger adults in peripheral vision than in central vision, due to the association of aging with higher losses of peripheral photoreceptors than of central photoreceptors (Gao \& Hollyfield, 1992).

Research Question 2: Do older adults show less precision in their motion estimates than young adults, and are these differences influenced by older adults' stereoacuity? Previous 
research has shown that aging is likely associated with increases in intraindividual variability (MacDonald et al., 2006), regardless of whether these differences are due to increases in neural noise or not. As previously mentioned, losses in stereoacuity have been found to predict FGMI exaggeration (Reed \& Burdett, 2002), and motion perception and stereoacuity may share neural mechanisms (Reed \& Burdett, 2002; Thompson \& Nawrot, 1999). Thus, it is hypothesized that the younger adults will show significantly higher precision (less variability) for their motion estimates in central and peripheral vision than both the older adult groups (i.e., the standard deviation of the motion estimates for the younger adults will be lower than for the older adult groups), and that the older adults with intact stereopsis will show significantly higher precision in central and peripheral vision than the older adults with poor stereopsis.

\section{Research Question 3: Is the difference in the magnitude of change in motion estimates} between central and peripheral vision different in younger and older adults, and are these differences influenced by older adults' stereoacuity? Increasing eccentricity is associated with decreases in photoreceptor density (Curcio, Sloan, Kalina, \& Hendrickson, 1990), decreases in $M$ (Strasburger et al., 2011), and increases in motion estimates for the FGMI task (Foster et al., 1981). Thus, it is expected that peripheral accuracy and precision will be poorer than central accuracy and precision, as reflected by higher values for peripheral accuracy and precision than for central accuracy and precision. Since aging results in higher losses in peripheral photoreceptors than in central photoreceptors (Gao \& Hollyfield, 1992), it is hypothesized that both older adult groups will show a larger difference between their central and peripheral vision than younger adults. However, it is unclear whether older adults with poor stereopsis will show a larger difference between their central and peripheral vision than older adults with intact stereopsis. Poor stereopsis could either increase or decrease the difference between central and 
peripheral vision performance. Stereoacuity losses may signal losses in neural mechanisms that support motion processing (Reed \& Burdett, 2002), which could have similar or differential effects on central and peripheral vision.

\section{Research Question 4: For older adults, are changes in age and stereoacuity associated} with changes in central and peripheral performance? Older age is associated with losses in stereoacuity (Lee \& Koo, 2005), and losses in stereoacuity have been found to predict FGMI exaggeration (Reed \& Burdett, 2002). Thus, it is hypothesized that older age and poorer stereoacuity (i.e., higher stereoacuity scores) will be associated with lower accuracy and precision in both central and peripheral performance. 


\section{Methods}

\section{Participants}

Fifty-six participants (34 younger adults with intact stereopsis, 12 older adults with intact stereopsis, 10 older adults with poor stereopsis) completed the FGMI task. Younger adults (ages 17-25) were students from Ryerson University recruited through the SONA Undergraduate Research Participant Pool; these students received one course credit for participating. Older adults (ages 65+) were recruited from the Ryerson Senior Participant Pool; these older adults received a $\$ 15$ honorarium for their participation.

Table 1 summarizes age and screening variables for the younger adults, older adults with intact stereopsis, and older adults with poor stereopsis. Age was compared between the two older adult groups. The younger adults were not included in these analyses, since the recruitment criteria ensured the younger adults were significantly younger than the older adults. For age, the results of the Kolmogorov-Smirnov test indicated that the assumption of normality was not violated for the older adults with intact stereopsis $(p>.05)$, but it was violated for the older adults with poor stereopsis $\left(\mathrm{D}_{10}=.30, p=.013\right)$. Thus, a Mann-Whitney U test was conducted to compare the older adult groups on age. The results of the Mann-Whitney U test indicated age was not significantly different between older adults with intact stereopsis and older adults with poor stereopsis, $U=58.00, p=.895$.

MoCA scores (an indicator of mild cognitive impairment) were also compared between the two older adult groups. The younger adults were not included in these analyses, since they are not considered at risk for mild cognitive impairment. For MoCA, the assumption of normality was not violated for either older adult group, according to the results of the Kolmogorov-Smirnov test ( $p$ s $>.05)$. The assumption of homogeneity of variances was also not 
violated, according to the results of the Levene's test $(p>.05)$. Thus, an independent-samples ttest was conducted to compare the older adult groups on MoCA scores. The independentsamples t-test indicated that MoCA scores were not significantly different between older adults with intact stereopsis and older adults with poor stereopsis, $t_{20}=.50, p=.622$.

All three groups were compared on corrected visual acuity. The assumption of normality was not tested for the older adults with intact stereopsis, as every participant in that group had 20/20 corrected visual acuity. The results of the Kolmogorov-Smirnov test indicated that the assumption of normality was violated for the younger adults $\left(\mathrm{D}_{34}=.20, p=.001\right)$ and older adults with poor stereopsis $\left(\mathrm{D}_{10}=.35, p=.001\right)$. Thus, a Kruskal-Wallis test was conducted to compare the three groups on corrected visual acuity. The results of the KruskalWallis test indicated a significant effect of group, $\chi^{2} 2, \mathrm{n}=56=20.57, p<.001$. Three MannWhitney U tests, with Bonferroni correction for multiple comparisons $(\alpha=.05 / 3=.017)$, showed that the younger adults had significantly better corrected visual acuity than older adults with intact stereopsis $(U=60.00, p<.001)$ and older adults with poor stereopsis $(U=68.00, p=$ .002); however, there was no significant difference in corrected visual acuity between the two older adult groups, $U=54.00, p=.507$. Nine of the younger adults, six of the older adults with intact stereopsis, and all ten of the older adults with poor stereopsis required vision correction. The level of correction ranged from -2.00 to 0.00 diopters for the younger adults, -2.50 to -0.50 diopters for the older adults with intact stereopsis, and -2.50 to 0.00 diopters for the older adults with poor stereopsis.

Stereoacuity (a measure of stereopsis), between the younger adults and older adults with intact stereopsis, was compared. The older adults with poor stereopsis were not included in these analyses, as the recruitment criteria required that they had poor stereopsis (60 seconds of arc or 
more). The results of the Kolmogorov-Smirnov test indicated that the assumption of normality was violated for both the younger adults $\left(\mathrm{D}_{34}=.54, p<.001\right)$ and older adults with intact stereopsis $\left(\mathrm{D}_{12}=.46, p<.001\right)$. Thus, a Mann-Whitney $\mathrm{U}$ test was conducted to compare the younger adults and older adults with intact stereopsis on stereoacuity. The results of the MannWhitney $U$ test indicated a trend towards higher stereoacuity in the younger adults than the older adults with intact stereopsis, $U=165.00, p=.070$. However, the two groups did not meaningfully differ on stereoacuity, given that all but two of the younger adults and all but three of the older adults had a stereoacuity of 40 seconds of arc, and that the criterion for intact stereoacuity made it possible only to have a stereoacuity of 40 or 50 seconds of arc as measured by the Circle Stereo test. The younger adults and older adults with intact stereopsis also had the same median values for stereoacuity. None of the participants showed eccentric fixation. Table 1.

Descriptive variables for participants

\begin{tabular}{lccc}
\hline & $\begin{array}{c}\text { Younger } \\
\text { adults } \\
(\mathrm{n}=34)\end{array}$ & $\begin{array}{c}\text { Older adults with } \\
\text { intact stereopsis } \\
(\mathrm{n}=12)\end{array}$ & $\begin{array}{c}\text { Older adults with } \\
\text { poor stereopsis } \\
(\mathrm{n}=10)\end{array}$ \\
\hline Age: $M d(M A D)$ & $18.00(0)$ & $73.00(4.00)$ & $70.00(3.50)$ \\
MoCA score: $\bar{x}(s)$ & $\mathrm{NA}$ & $26.75(2.42)$ & $27.20(1.62)$ \\
Corrected visual acuity: $M d(M A D)$ & $20 / 16(3.50)^{\mathrm{a}}$ & $20 / 20(0)^{\mathrm{b}}$ & $20 / 20(0)^{\mathrm{b}}$ \\
Stereoacuity: $M d(M A D)$ & $40.00(0)$ & $40.00(0)$ & $90.00(10.00)$ \\
\hline
\end{tabular}

For each variable, groups with different letters were significantly different from each other at $p<$ .05. Median absolute deviation $(M A D)=\operatorname{median}\left[\left|X_{i}-\operatorname{median}\left(X_{i}\right)\right|\right]$.

\section{Measures}

Visual and medical history. Participants completed a visual and medical history questionnaire. For older adults, the Older Adult: Visual and Medical History Survey (Appendix A) was administered during the recruitment process to avoid booking older participants who may not have been eligible to participate in this study. Younger adults completed the Younger Adult: 
Visual and Medical History Survey (Appendix B). Participants had their vision tested within the last 2 years, and provided any history of visual disorders (e.g., strabismus) or medical conditions known to affect vision (e.g., diabetes). None of the participants had any of these conditions. Older adults with dementia, multiple sclerosis, age-related macular degeneration, untreated glaucoma, and/or central dense cataracts were not eligible to participate in the study.

Cognitive assessment. The Montreal Cognitive Assessment Version 1 (MoCA; Nasreddine et al., 2005; Appendix C), a 10-minute cognitive screening tool, was used as an indicator of overall cognitive function. During this task, the experimenter read each question of the assessment to the participant. The experimenter recorded the participants' responses for each question. Early studies suggested a cut-off score of less than 26 could be used as a screening tool for potential mild cognitive impairment (Nasreddine et al., 2005). However, the findings of some recent studies have questioned the validity of this cut-off score. In a large ethnically diverse sample, older adults between the ages of 65 and 75 had a mean score of 22.05 with a standard deviation of 4.48 (Rossetti, Lacritz, Cullum, \& Weiner, 2011). Similarly, in a study of seniors living in a rural Canadian community, the mean score was 21.25 with a standard deviation of 4.57 (Dolinar, Pleta, Salmoni, \& Johnson, 2016).

Stereoacuity. Stereopsis was assessed using a test for stereoacuity, the Titmus Circle Stereo Test (Stereo Optical Co. Inc; Figure 1). Using both eyes, participants viewed nine sets of four circles through polarized glasses. Each circle is composed of two overlapping circle images. Of the four circles in each set, one of the circles has two circle images that do not quite overlap. In participants with functioning stereopsis, this disparity causes the image of a circle to pop out from the background. For each set of four circles, the participant was asked to identify the circle that appeared to pop out from the background. The disparity between the two circles decreases 
in each set, decreasing the amount of pop out as the participant progresses from the first set to the ninth set. Intact stereopsis was defined as 50 seconds of arc or less (i.e., being able to identify the correct circle at the eighth or ninth set), and poor stereopsis was defined as 60 seconds of arc or more (i.e., not being able to identify the correct circle beyond the seventh set).

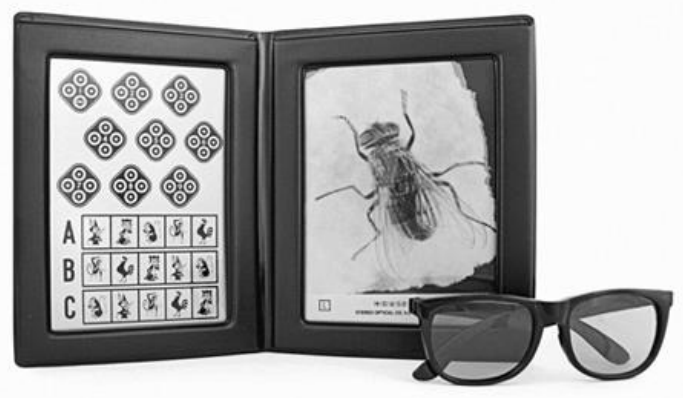

Figure 1. Circle Stereo test (Picture credit: Precision Vision, 2017)

Visual acuity. Visual acuity was assessed monocularly in each participant's right eye using an eye patch covering the left eye and the $96 \%$ contrast (black on white) Regan Eye Chart (Paragon Services Inc; Figure 2). Seated $10 \mathrm{ft}$. from the chart participants read rows of letters that gradually decreased in size with each row. The smallest row of letters that could be successfully read by the participant (i.e., making fewer than two mistakes in that row) was used to determine that participant's acuity score. This score was recorded and converted to a standardized score representing what the participant could read accurately at $20 \mathrm{ft}$. To participate in the study, younger adults were required to have normal or corrected-to-normal visual acuity of $20 / 20$ or better, which means that these participants should be able to read at 20 $\mathrm{ft}$. what a person with normal vision could read at $20 \mathrm{ft}$. Older adults were not required to meet a specific level of normal or corrected-to-normal visual acuity, but their acuity was recorded to assess its potential impact on their performance. 


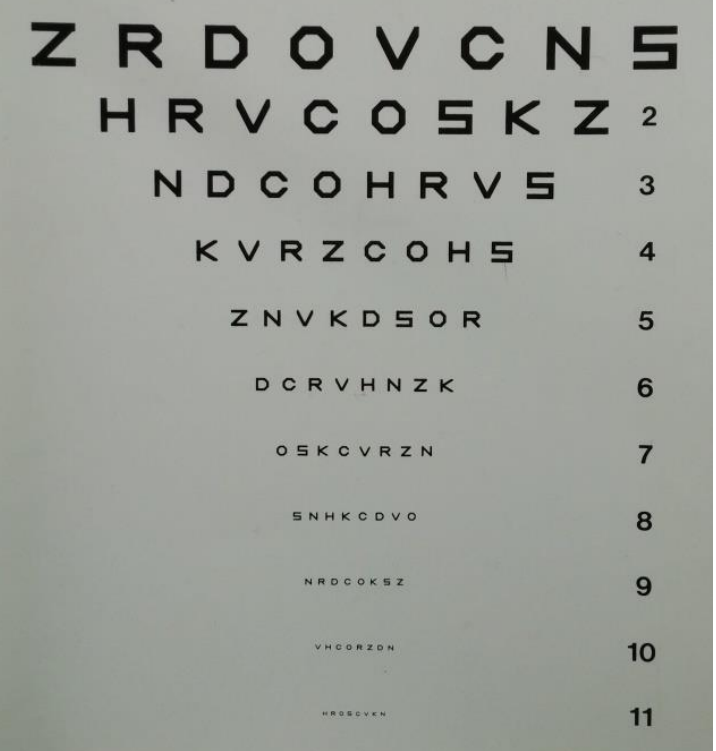

Figure 2. Regan Eye Chart (96\% contrast)

Eccentric fixation. Eccentric fixation, the use of a retinal area eccentric to the fovea for fixation, was assessed monocularly in each participant's right eye using Maxwell's Spot (i.e., the perception of a reddish spot produced by alternate viewing of a brightly illuminated surface through neutral/yellow and blue/purple filters; Isobe \& Motokawa, 1955). The presentation of white/yellow light results in differential adaptation of the short-wave-sensitive cones in the foveola than in the rest of the macula, since fewer short-wave-sensitive cones are found in the fovea. Subsequent presentation of blue/purple light results in a reduced response in the foveola, producing the perception of a reddish spot afterimage in the centre of the fovea (Loughman, Davison, Nolan, Akkali, \& Beatty, 2010). Participants were seated $28.5 \mathrm{~cm}$ away from the screen, and wore an eye patch covering the left eye. Participants were instructed to fixate on a yellow screen for $10 \mathrm{~s}$. Following this, a blue screen with a black fixation cross was presented. Participants were instructed to focus on the centre of the fixation cross, and to use an adhesive arrow to identify where on the screen they perceived the centre of Maxwell's Spot (Figure 3). 
The level of eccentric fixation was measured by the direction and distance in mm that Maxwell's Spot appeared from the centre of the fixation cross (e.g., $2 \mathrm{~mm}$ to the right of fixation).

A

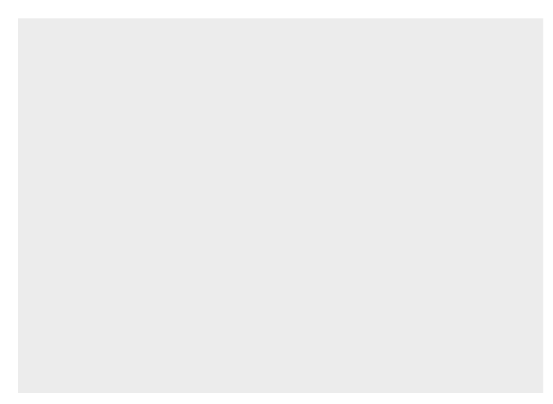

B

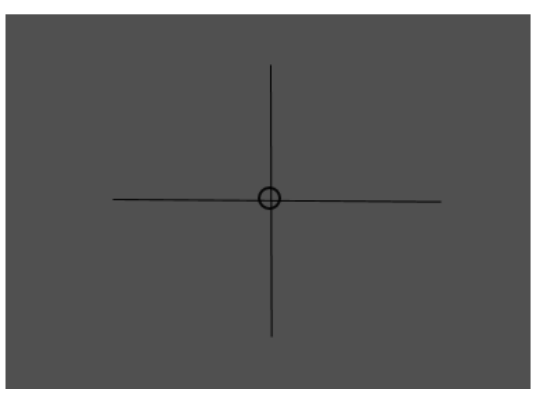

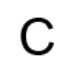

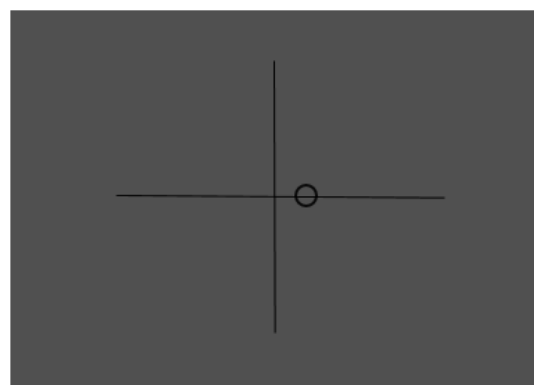

Figure 3. Perception of Maxwell's Spot. A yellow screen (A) was presented for $10 \mathrm{~s}$ followed by a blue screen with a fixation cross. For foveal fixators, Maxwell's Spot would appear in the centre of the fixation cross (B). For eccentric fixators, Maxwell's Spot would appear some distance from the centre of the fixation cross $(C)$.

FGMI task. Participants were comfortably seated $228 \mathrm{~cm}$ from a monitor on which the FGMI task (Figure 4) was presented. The FGMI task was programmed using EPrime 2.0 (Psychology Software Tools, Pittsburgh, PA). Participants completed this task using their right eye while their left eye was covered. Two $2 \mathrm{~mm}$ ( 8 pixel) white dots separated by $2 \mathrm{~mm}$, from the centre of the first dot to the centre of the second dot, were presented on a black background. At the beginning of each trial, the first dot was presented for $15 \mathrm{~ms}$, followed by a blank screen for $40 \mathrm{~ms}$. Then, the second dot was flashed for $15 \mathrm{~ms}$. After a 2 second delay, a horizontal standard line was presented [randomly 0.05 degrees ( 4 pixels) or 0.5 degrees ( 80 pixels) in length]. Participants indicated whether the line presented was longer or shorter than the distance perceived to have been travelled by the dot. The length of the line was then modified for the next presentation of the dots according to a staircase procedure (Reed \& Burdett, 2002). If the participant indicated they perceived the linear extent of the illusion was shorter than the line 
length, the line length was halved for the next presentation of the dots. If the participant indicated they perceived the linear extent of the illusion was longer than the line length, the line length was doubled for the next presentation of the dots. The line length was increased or decreased in this manner until the participant changed direction (e.g., changed from indicating that the perceived linear extent was longer than the line length to indicating that the perceived linear extent was shorter than the line length). On subsequent presentations, the line length was halfway between the last longer than response and last shorter than response. The staircase procedure ended when the change in length of a new line was less than one pixel different from a previous line. The perceived linear extent of the illusion was taken as halfway between these two line lengths. FGMI exaggeration was defined as perceived linear extents greater than the actual $2 \mathrm{~mm}$ ( 8 pixel) distance between the two dots. This staircase procedure was completed 16 times for each participant. One block of 8 trials was completed centrally (at $0^{\circ}$ visual angle), and one block of 8 trials was completed peripherally at $30^{\circ}$ visual angle from the fovea (i.e., in the right temporal peripheral field), a distance the findings of Foster et al. (1981) suggest should show maximal exaggeration. The order of central and peripheral trials was counterbalanced between participants. Accuracy and precision were calculated by taking the mean and standard deviation, respectively, of the eight central and eight peripheral trials of the linear extent estimates. Accuracy and precision were calculated separately for the central and peripheral trials, resulting in four values: (1) central vision accuracy, (2) central vision precision, (3) peripheral vision accuracy, and (4) peripheral vision precision. 


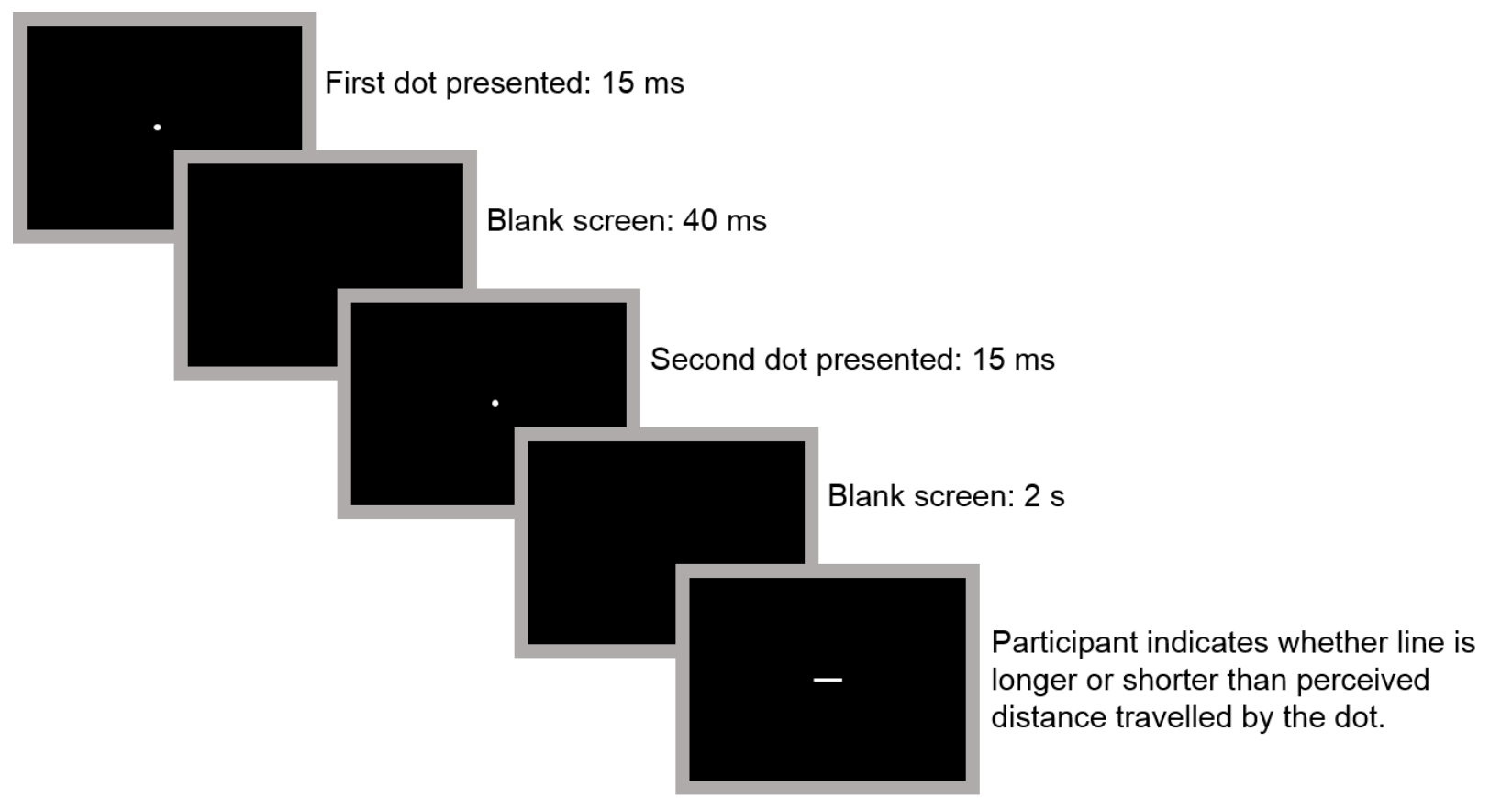

Figure 4. Sample presentation of the dots and a standard line at the beginning of each trial for the FGMI task. After each response by the participant, the dots were presented again and the line was adjusted in length following the staircase procedure described in the FGMI task section. Each trial ended when the change in line length would be less than 1 pixel different from a previous line length.

\section{Procedure}

Prior to coming into the lab, older participants were guided through the Older Adult: Visual and Medical History Survey (Appendix A) over the telephone to determine eligibility. This survey required approximately five minutes to complete and only eligible older adults were invited to participate in the study. Upon arriving at the lab, participants were seated and given a consent form that described the details of the study. The experimenter reviewed the consent form with the participant. Participants were asked to read and sign the consent form before beginning the experiment. After signing the consent form, older adults were compensated with $\$ 15$ for participating while younger adults were compensated with course credit. Younger adults 
completed the Younger Adult: Visual and Medical History Survey (Appendix B), which required fewer than five minutes to complete.

The experiment required one hour to complete. During this time, participants completed several tasks. First, older participants completed Version 1 of the MoCA (approximately 10 minutes). Following this, participants completed the Titmus Circle Stereo Test (Stereo Optical Co. Inc; approximately five minutes). Then, participants completed the visual acuity test (approximately five minutes). Participants were tested for eccentric fixation using Maxwell's Spot (approximately five minutes). Finally, each participant completed the FGMI task (approximately 30 minutes). Although participants could ask questions during the study, feedback on tasks was not provided until the end of the experiment when the participant was debriefed regarding the purpose of the study.

\section{Statistical Analyses}

All statistical analyses were conducted in SPSS 22 (see Appendix D for statistical summaries). Before testing whether FGMI exaggeration occurred in each group in both central and peripheral vision [i.e., whether average estimates of motion were greater than the $2 \mathrm{~mm}$ ( 8 pixel) distance between the centre of the first dot and the centre of the second dot], KolmogorovSmirnov tests were conducted to assess normality. One-sample t-tests were conducted when the assumption of normality was not violated, while one-sample Wilcoxon signed rank tests were conducted when the assumption of normality was violated. To determine the magnitude of the differences between central and peripheral accuracy estimates and central and peripheral precision estimates, difference scores were calculated (vision accuracy difference $=$ peripheral vision accuracy - central vision accuracy; vision precision difference $=$ peripheral vision precision - central vision precision). Prior to assessing the effect of group on accuracy, 
precision, and difference scores, Kolmogorov-Smirnov tests were conducted to assess normality. If the assumption of normality was not violated, Levene's test was conducted to assess homogeneity of variances. Because these data violated the assumptions of normality and/or homogeneity of variances, Kruskal-Wallis tests and Mann-Whitney U post-hoc tests were conducted. Effect size ( $r$ ) for the Mann-Whitney U post-hoc tests were calculated by dividing the z-statistic by the square root of the total sample size (Rosenthal, 1991). Finally, Spearman rank-order correlations were conducted to assess the relationships of age and stereoacuity with accuracy and precision within the older adults. $r$ and $\rho$ values of $0.2,0.5$, and 0.8 were considered to reflect small, moderate, and strong effects, respectively (Ferguson, 2009). 


\section{Results}

Research Question 1: Do older adults show less accuracy in their motion estimates than young adults, and are these differences influenced by older adults' stereoacuity?

The FGMI task required individuals to estimate the distance perceived to have been travelled by the dot. Each participant's accuracy scores represent the mean of their linear extent estimates, as calculated separately for the central and peripheral trials. It was hypothesized that both younger adults and older adults with intact stereopsis would show significantly higher accuracy in central and peripheral vision than the older adults with poor stereopsis [i.e., the estimates of the younger adults and older adults with intact stereopsis would be lower, and therefore closer to the actual $(2 \mathrm{~mm})$ distance between the centre of the first dot and the centre of the second dot, than the estimates of the older adults with poor stereopsis], and that younger adults and older adults with intact stereopsis would not significantly differ on accuracy in central and peripheral vision. Prior to conducting analyses on central accuracy and precision, one older adult with intact stereopsis was removed, due to missing central vision trials.

For central vision accuracy, the results of the Kolmogorov-Smirnov test indicated all three groups were normally distributed $(p s>.05)$. Thus, one-sample t-tests were conducted to determine whether each group showed FGMI exaggeration. The results of the one-sample t-tests indicated that all three groups showed FGMI exaggeration ( $p \mathrm{~s} \leq .05$; see Appendix D). Although the assumption of normality was not violated, the results of the Levene's test indicated the assumption of homogeneity of variances was violated, $W_{2,52}=7.58, p=.001$. Thus, a KruskalWallis test was conducted to compare the groups on central vision accuracy. The results of the Kruskal-Wallis test indicated a trend towards a significant effect of group, $\chi_{2, \mathrm{n}}^{2}=55=5.45, p=$ .066. Three Mann-Whitney U tests, with Bonferroni correction for multiple comparisons ( $\alpha=$ 
$.05 / 3=.017$ ), showed no significant difference in central vision accuracy between the younger adults $(M d=17.31, n=34)$ and the older adults with intact stereopsis $(M d=10.88, n=11), U=$ $164.50, p=.552, r=.08$. However, there was a trend towards higher central vision accuracy in younger adults than in older adults with poor stereopsis $(M d=33.44, n=10), U=94.00, p=$ $.033, r=.29$. There was also a trend towards higher central vision accuracy in older adults with intact stereopsis than in older adults with poor stereopsis, $U=27.00, p=.049, r=.27$. Medians and median absolute deviations for central vision accuracy for the younger adults, older adults with intact stereopsis, and older adults with poor stereopsis are presented in Figure 5.

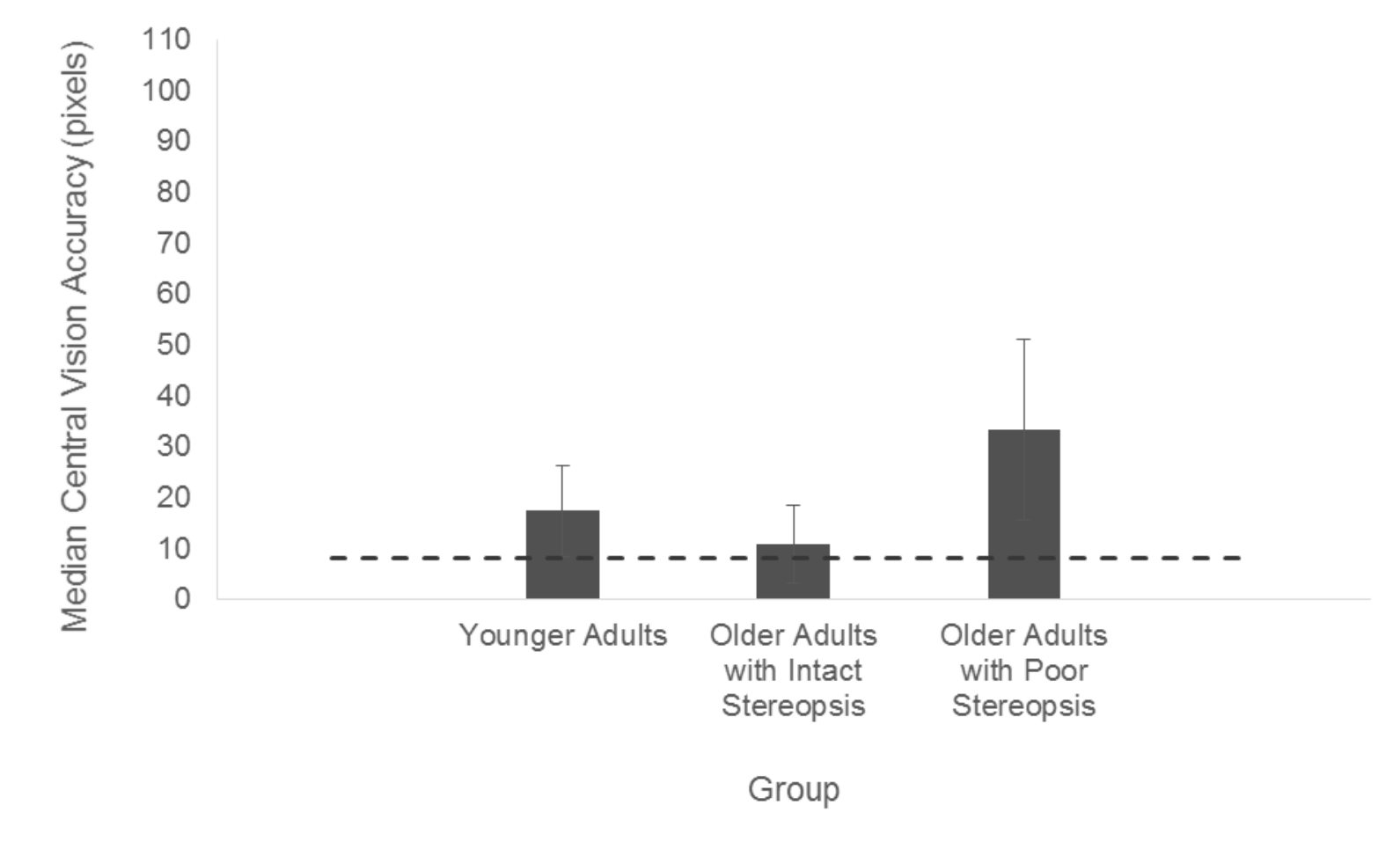

Figure 5. Median central vision accuracy for the younger adults, older adults with intact stereopsis, and older adults with poor stereopsis. Error bars represent median absolute deviations. Dashed line represents the distance of 8 pixels from the centre of the first dot to the centre of the second dot that, in the absence of exaggeration, would be perceived to be travelled by a single dot in the apparent motion display. 
For peripheral vision accuracy, the results of the Kolmogorov-Smirnov test indicated the assumption of normality was not violated for the older adults with intact stereopsis $\left(\mathrm{D}_{12}=.14, p\right.$ $=.200)$, but it was violated for the younger adults $\left(\mathrm{D}_{34}=.16, p=.030\right)$ and older adults with poor stereopsis $\left(\mathrm{D}_{10}=.27, p=.042\right)$. Thus, to determine whether each group showed FGMI exaggeration, a one-sample t-test was conducted for the older adults with intact stereopsis, while one-sample Wilcoxon signed rank tests were conducted for the younger adults and older adults with poor stereopsis. The results of these tests indicated that all three groups showed FGMI exaggeration $(p s<.05)$. The results of the Levene's test indicated the assumption of homogeneity of variances was violated, $W_{2,53}=7.56, p=.001$. Thus, a Kruskal-Wallis test was conducted to compare the three groups on peripheral vision accuracy. The results of the Kruskal-Wallis test indicated a trend towards a significant effect of group, $\chi^{2} 2, \mathrm{n}=56=4.88, p=$ .087. Three Mann-Whitney U tests, with Bonferroni correction for multiple comparisons ( $\alpha=$ $.05 / 3=.017$ ), showed no significant difference in peripheral vision accuracy between the younger adults $(M d=29.41, n=34)$ and the older adults with intact stereopsis $(M d=37.02, n=$ 12), $U=186.00, p=.653, r=.06$. However, there was a trend towards higher peripheral vision accuracy in younger adults than in older adults with poor stereopsis ( $M d=69.71, n=10), U=$ $96.00, p=.038, r=.28$. There was also a trend towards higher peripheral vision accuracy in older adults with intact stereopsis than in older adults with poor stereopsis, $U=32.00, p=.065, r$ $=.25$. Medians and median absolute deviations for peripheral vision accuracy for the younger adults, older adults with intact stereopsis, and older adults with poor stereopsis are presented in Figure 6. 


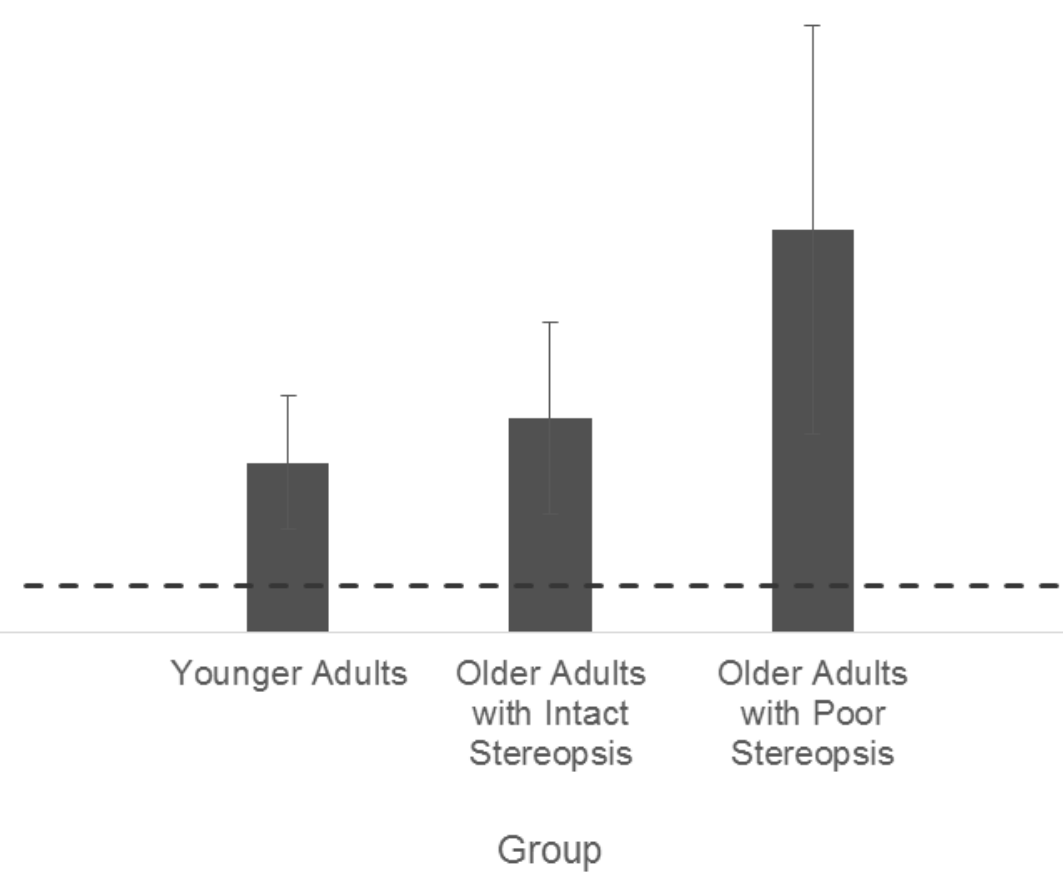

Figure 6. Median peripheral accuracy for the younger adults, older adults with intact stereopsis, and older adults with poor stereopsis. Error bars represent median absolute deviations. Dashed line represents the distance of 8 pixels from the centre of the first dot to the centre of the second dot that, in the absence of exaggeration, would be perceived to be travelled by a single dot in the apparent motion display.

\section{Research Question 2: Do older adults show less precision in the linear extent of their} movement estimates than young adults, and are these differences influenced by older adults' stereoacuity?

Each participant's precision scores represent the standard deviation of their linear extent estimates, as calculated separately for the central and peripheral trials. It was hypothesized that the younger adults would show significantly higher precision (less variability) for their linear extent estimates in central and peripheral vision than both the older adult groups (i.e., the standard deviation of the estimates of the younger adults would be lower than that of the 
estimates of the older adult groups), and that the older adults with intact stereopsis would show significantly higher precision in central and peripheral vision than the older adults with poor stereopsis.

For central vision precision, the results of the Kolmogorov-Smirnov test indicated that the assumption of normality was not violated for the older adult groups ( $p s>.05)$, but it was violated for the younger adults $\left(\mathrm{D}_{34}=.19, p=.003\right)$. The results of the Levene's test indicated the assumption of homogeneity of variances was violated, $W_{2,52}=5.17, p=.009$. Thus, a Kruskal-Wallis test was conducted to compare the three groups on central vision precision. The results of the Kruskal-Wallis test indicated no significant differences in central vision precision between the younger adults $(M d=8.62, n=34)$, older adults with intact stereopsis $(M d=6.28, n$ $=11)$, and older adults with poor stereopsis $(M d=11.95, n=10), \chi^{2} 2, \mathrm{n}=55=3.17, p=.205$. Medians and median absolute deviations for central vision precision for the younger adults, older adults with intact stereopsis, and older adults with poor stereopsis are presented in Figure 7. 


\section{Group}

Figure 7. Median central vision precision for the younger adults, older adults with intact stereopsis, and older adults with poor stereopsis. Error bars represent median absolute deviations.

For peripheral vision precision, the assumption of normality was not violated for the older adult groups $(p s>.05)$, but it was violated for the younger adults, $\left(\mathrm{D}_{34}=.24, p<.001\right)$. The results of the Levene's test indicated the assumption of homogeneity of variances was violated, $W_{2,53}=4.79, p=.012$. Thus, a Kruskal-Wallis test was conducted to compare the three groups on peripheral vision precision. The results of the Kruskal-Wallis test indicated no significant differences in peripheral vision precision between the younger adults $(M d=16.07, n$ $=34)$, older adults with intact stereopsis $(M d=12.25, n=12)$, and older adults with poor stereopsis $(M d=22.12, n=10), \chi^{2} 2, \mathrm{n}=56=2.01, p=.366$. Medians and median absolute deviations for peripheral vision precision for the younger adults, older adults with intact stereopsis, and older adults with poor stereopsis are presented in Figure 8. 


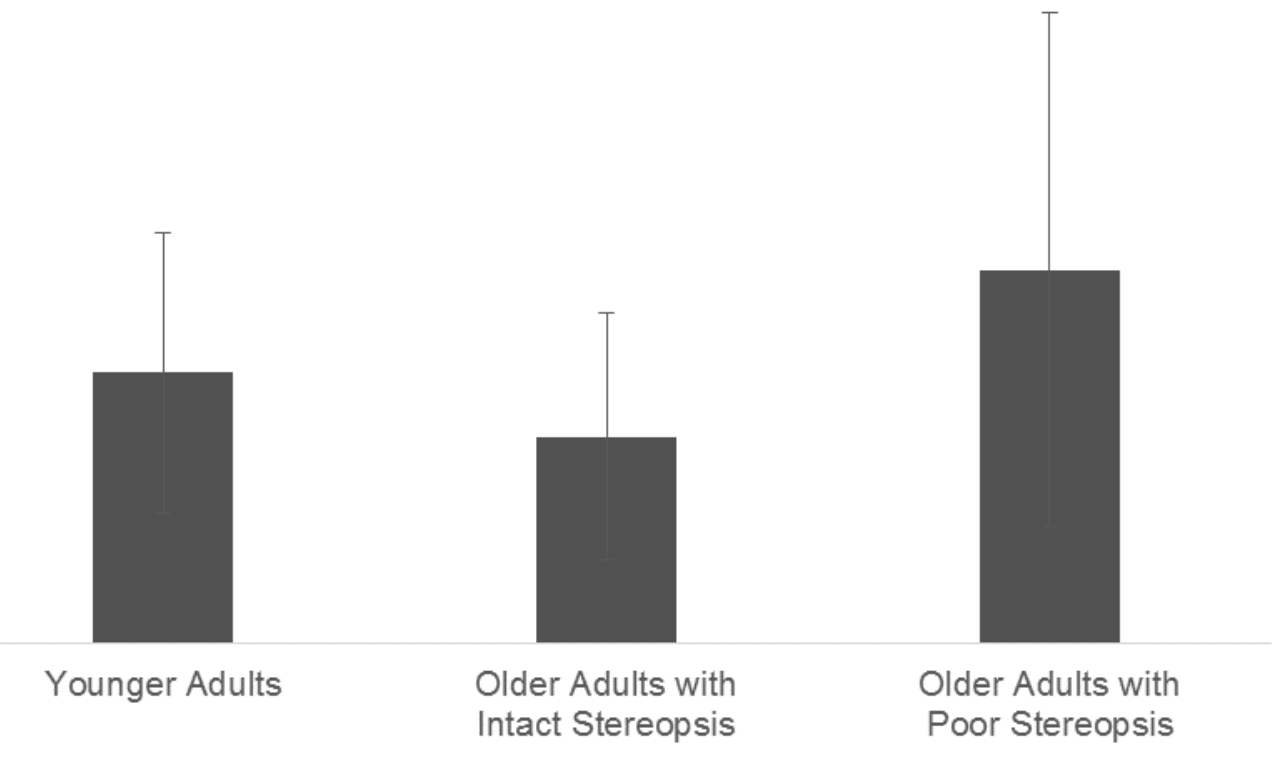

Group

Figure 8. Median peripheral vision precision for the younger adults, older adults with intact stereopsis, and older adults with poor stereopsis. Error bars represent median absolute deviations.

\section{Research Question 3: Is the difference in the magnitude of change in motion estimates between central and peripheral vision different in younger and older adults, and are these differences influenced by older adults' stereoacuity?}

To examine the magnitude of the differences between central and peripheral vision in accuracy and precision by group (younger adults, older adults with intact stereopsis, older adults with poor stereopsis), difference scores were calculated for vision accuracy (peripheral vision accuracy - central vision accuracy) and vision precision (peripheral vision precision - central vision precision). For the vision accuracy difference scores, the assumption of normality was not violated for the younger adults and older adults with intact stereopsis ( $p \mathrm{~s}>.05)$, but it was violated for older adults with poor stereopsis $\left(\mathrm{D}_{10}=.39, p<.001\right)$. The results of the Levene's 
test indicated the assumption of homogeneity of variances was violated, $W_{2,52}=3.78, p=.029$. Thus, a Kruskal-Wallis test was conducted to compare the groups on vision accuracy difference scores. The results of the Kruskal-Wallis test indicated a trend towards a significant effect of group, $\chi^{2} 2, \mathrm{n}=55=4.62, p=.100$. Three Mann-Whitney $\mathrm{U}$ tests, with Bonferroni correction for multiple comparisons $(\alpha=.05 / 3=.017)$, showed no significant difference in vision accuracy difference scores between the younger adults $(M d=8.78, n=34)$ and the older adults with intact stereopsis $(M d=15.67, n=11), U=135.00, p=.170, r=.19$. There was also no significant difference in vision accuracy difference scores between the older adults with intact stereopsis and the older adults with poor stereopsis $(M d=26.88, n=10), U=46.00, p=.526, r=.09$. However, there was a trend towards higher vision accuracy difference scores in the older adults with poor stereopsis than the younger adults, $U=102.00, p=.057, r=.26$. Medians and median absolute deviations for the vision accuracy difference scores for the younger adults, older adults with intact stereopsis, and older adults with poor stereopsis are presented in Figure 9. 

30 $-5$

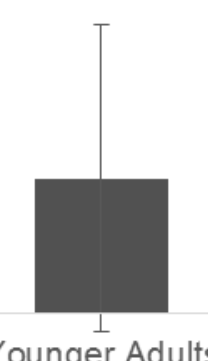

Younger Adults

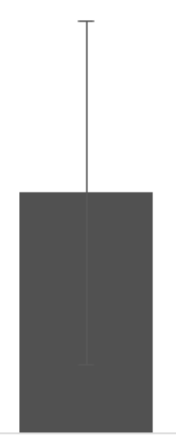

Older Adults with Intact Stereopsis

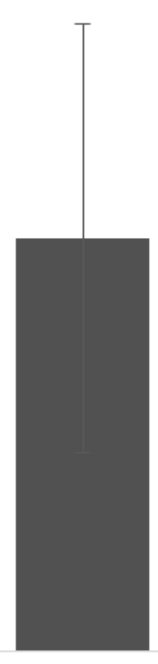

Older Adults with Poor Stereopsis

Group

Figure 9. Median vision accuracy difference scores (peripheral vision accuracy - central vision accuracy) for the younger adults, older adults with intact stereopsis, and older adults with poor stereopsis. Error bars represent median absolute deviations.

For the vision precision difference scores, the assumption of normality was not violated for the older adult groups ( $p$ s $>.05)$, but it was violated for the younger adults $\left(\mathrm{D}_{34}=.24, p<\right.$ .001). The results of the Levene's test indicated the assumption of homogeneity of variances was not violated, $W_{2,52}=2.22, p=.119$. Since the assumption of normality was violated, a Kruskal-Wallis test was conducted to compare the groups on vision precision difference scores. The results of the Kruskal-Wallis test indicated no significant differences in vision precision difference scores between the younger adults $(M d=4.90, n=34)$, older adults with intact stereopsis $(M d=5.21, n=11)$, and older adults with poor stereopsis $(M d=7.28, n=10), \chi^{2} 2, \mathrm{n}=$ $55=1.14, p=.566$. Medians and median absolute deviations for the vision precision difference 
scores for the younger adults, older adults with intact stereopsis, and older adults with poor stereopsis are presented in Figure 10.

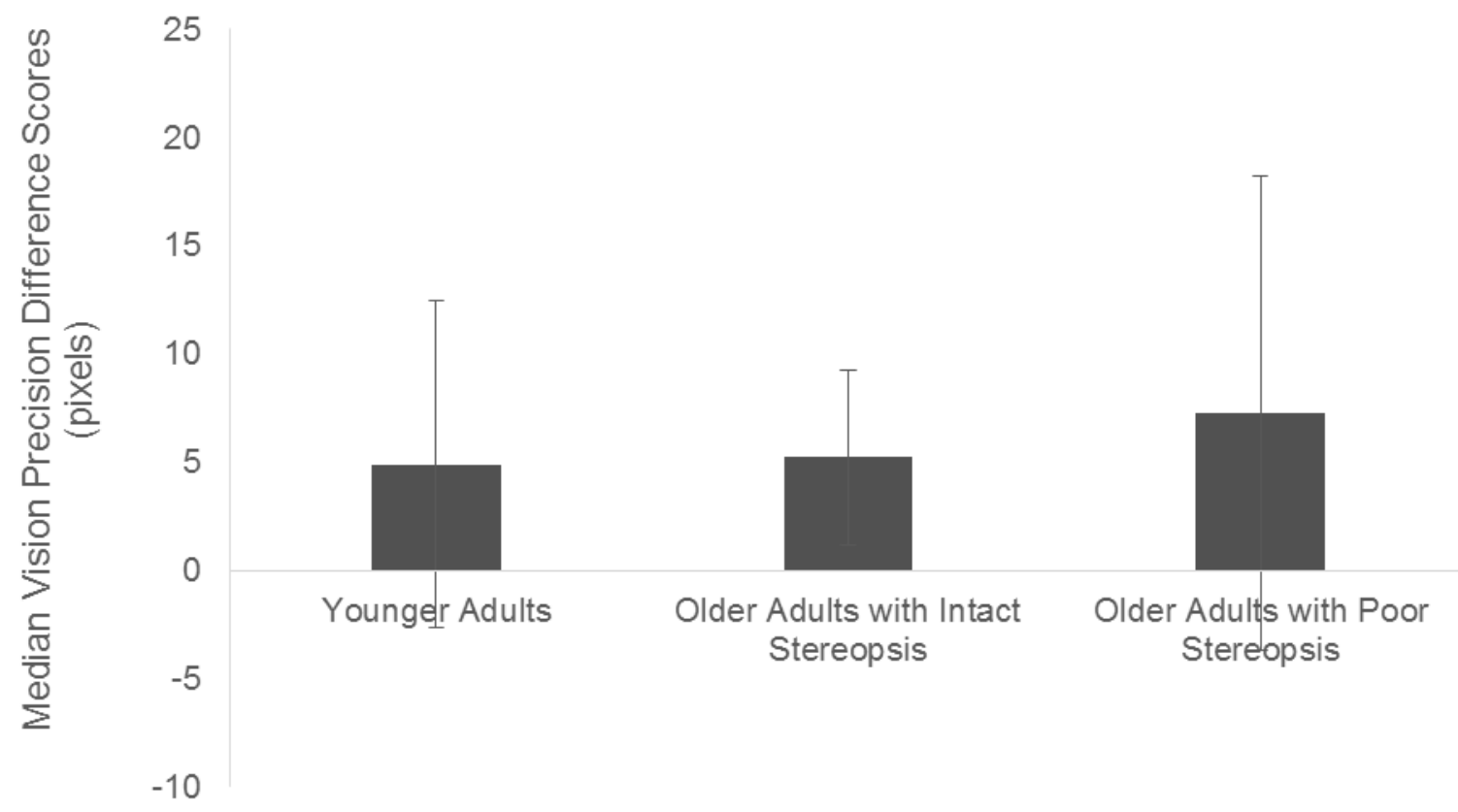

Group

Figure 10. Median vision precision difference scores (peripheral vision precision - central vision precision) for the younger adults, older adults with intact stereopsis, and older adults with poor stereopsis. Error bars represent median absolute deviations.

\section{Research Question 4: For older adults, are changes in age and stereoacuity associated with changes in central and peripheral performance?}

Given that the earlier group comparisons on central and peripheral performance were conducted on rank-order data, Spearman rank-order correlations were conducted to evaluate the associations of age and stereoacuity with central and peripheral performance across the older adults. For stereoacuity, higher values indicated poorer performance. It was hypothesized that higher age and poorer stereoacuity would be associated with lower accuracy and precision in both central and peripheral performance. Changes in corrected visual acuity were not expected to 
be associated with changes in central or peripheral performance, as none of the participants had corrected visual acuity below 20/25. Poorer stereoacuity was associated with poorer peripheral vision accuracy $\left(\rho_{20}=.47, p=.028\right)$. There were also trends between poorer stereoacuity and poorer central vision accuracy $\left(\rho_{19}=.43, p=.053\right)$ and poorer peripheral vision precision $\left(\rho_{20}=\right.$ $.40, p=.063)$. Stereoacuity was not associated with central vision precision $\left(\rho_{19}=.34, p=\right.$ .137). Age was not associated with central vision accuracy $\left(\rho_{19}<.01, p=.996\right)$, central vision precision $\left(\rho_{19}=-.27, p=.230\right)$, peripheral vision accuracy $\left(\rho_{20}=-.13, p=.561\right)$, and peripheral vision precision $\left(\rho_{20}=-.03, p=.894\right)$. 


\section{Discussion}

To examine the role of stereoacuity losses in older adults' performance on the FGMI task, the accuracy and precision of younger adults, older adults with intact stereopsis, and older adults with poor stereopsis were compared for central and peripheral vision. The results of the present research indicated: (1) age alone was not associated with differences in central or

peripheral accuracy or precision; and (2) stereoacuity losses in older adults were associated with poorer central and peripheral accuracy and poorer peripheral precision, but not poorer central precision. These findings suggest stereopsis losses in the context of aging may signal neural or oculomotor changes, resulting in reductions in positional accuracy that could account for some findings of motion perception deficits with aging.

\section{Relationships between Aging Alone and Central and Peripheral Accuracy and Precision of Motion Estimates}

The findings of no differences in central or peripheral accuracy of motion estimates between the younger adults and older adults with intact stereopsis, and of no correlation between age and central or peripheral accuracy of estimates, suggest that age alone is not responsible for the lower central and peripheral accuracy of the older adults with poor stereopsis. There is substantial variability in the extent to which individuals experience age-related deficits in function, in large part due to genetic and environmental differences between individuals (Brooks-Wilson, 2013; Lin et al., 2012). However, aging is associated with losses in stereoacuity (Lee \& Koo, 2005), and losses in stereoacuity have been associated with greater FGMI exaggeration (Reed \& Burdett, 2002). Thus, at the very least, aging makes an individual more likely to have the stereoacuity deficits that were associated with lower central and peripheral accuracy on the FGMI task. In this study, it was only older adults with stereopsis losses that showed greater 
FGMI exaggeration than younger adults in central and peripheral accuracy of estimates for the FGMI task. However, without a group of younger adults with poor stereopsis but otherwise normal vision, it is difficult to say with more certainty that aging did not contribute to these findings of poorer central and peripheral accuracy in older adults with poor stereopsis. It was not possible to include a group of younger adults with poor stereopsis, since stereopsis losses in younger adults are typically associated with confounding developmental visual disorders (e.g., strabismus) that would make them systematically different from the other three groups on variables other than age and stereoacuity (see Reed \& Burdett, 2002). Thus, the findings of the present study cannot rule out definitively that changes associated with aging did not interact with changes signaled by stereopsis losses.

There are several potential changes with aging that could interact with the changes signaled by stereopsis losses. Leat and colleagues (2013), for example, found nearly half of older adults over the age of 60 had disorders of binocular vision and eye movement, which could have negative effects on motion perception through their effects on visual abilities that support motion perception (e.g., fixation stability). Aging is also associated with losses in visual acuity, which if not adequately corrected for, could affect motion perception, since adequate visual acuity is needed to bring objects into focus. Losses in visual acuity likely did not contribute to any of the findings in the present study, since visual acuity was adequately corrected in all participants. It is also possible that the effects of aging, in the presence or absence of stereopsis losses, may become apparent in a group of older adults of more advanced age. Visual deficits associated with aging are generally more pronounced with advanced age. Lee and Koo (2005) found the greatest stereoacuity losses in participants over the age of 70, while Leat and colleagues (2013) found the highest percentage of disorders of binocular vision or eye movement in the $80+$ age 
group. Thus, a group of oldest-old adults (ages $80+$ ) would likely have greater stereoacuity losses. What is not clear is whether exaggerated FGMI perception only becomes apparent in the oldest-old adults who have stereoacuity losses. If oldest-old adults with and without stereoacuity losses showed FGMI exaggeration, this finding would suggest that advanced age alone is associated with lower accuracy of motion estimates. If only oldest-old adults with stereoacuity losses showed FGMI exaggeration, this finding would suggest that stereopsis losses must be present in advanced aging before accuracy of motion estimates is negatively affected.

Many previous researchers have found aging is associated with higher intraindividual variability in behaviour and neural activity during task performance (see MacDonald et al., 2006). Early proponents of the neural noise hypothesis posited that increases in neural noise with aging explained behavioural findings suggestive of age-related deficits in information processing (Gregory, 1957), while more recent proponents have extended this idea to explain variability in neuroimaging measures (e.g., EEG, BOLD) as well (MacDonald, Li, \& Bäckman, 2009). Another possible explanation for these increases in intraindividual variability are shifts in strategies during tasks, or to adaptive mechanisms that maintain performance in the context of aging (Garrett et al., 2010; Garrett et al., 2013). The findings of a recent EEG study indicated that increases in $1 / f$ noise with aging predicted poorer performance on a visual working memory task (Voytek et al., 2015). This finding suggests that aging is associated with increases in 1/f noise for EEG, and these increases may negatively affect visual working memory performance. However, using BOLD imaging, Garrett and colleagues (2010) found brain areas where older adults had more variability than younger adults, but older adults had lower variability overall across the brain, which implies the relationship between neural noise, age, and brain function is not as simple as higher neural noise with aging reflects poorer brain function. Garrett and 
colleagues (2013) also suggest that increases in neural noise could be an adaptive mechanism to protect function with aging, since neural connections formed in noise are more resistant to disruption. Thus, the physiological relevance of increases and decreases in noise with aging for different types of neural measures and across different brain regions remain to be further clarified.

In the context of this literature, the lack of relationship between aging and central or peripheral precision of motion estimates in the group and correlational analyses runs contrary to previous findings of higher intraindividual variability with aging and to the behavioural predictions of the neural noise hypothesis and other theories related to increasing intraindividual variability with aging. A potential explanation for this discrepancy between the findings of the present study and the literature is that the within-group variability in motion estimates for the FGMI task was too high in all three groups to detect higher precision in the younger adults relative to the two older adult groups. However, the median of the precision values for the older adults with intact stereopsis were lower (i.e., better) than in the younger adults for both central and peripheral motion estimates, which similarly suggests aging alone was not associated with poorer precision. It is also possible that the sample did not include enough older adults of advanced age to find a correlation between aging and lower central or peripheral precision in the correlational analyses within the older adult sample, since the sample had only four oldest-old adults ( 1 with intact stereopsis, 3 with poor stereopsis). However, given the correlations of age with central and peripheral precision across the older adults were small, not significant, and in the opposite direction, it is unlikely that the inclusion of more oldest-old adults would have produced a correlation between aging and central or peripheral precision. As discussed earlier in the context of accuracy, it is possible that a group of oldest-old adults with and without 
stereoacuity losses could show the same pattern of results or a different pattern of results as the older adults in this study. If oldest-old adults with and without stereoacuity losses did not show lower precision, this finding would suggest that advanced age is not associated with lower precision, regardless of whether stereoacuity losses are present or not. If oldest-old adults with and without stereoacuity losses showed similarly lower precision, this finding would suggest that advanced age alone is associated with lower precision. If only oldest-old adults with stereoacuity losses showed lower precision, this finding would suggest that stereopsis losses must be present in advanced aging before precision is negatively affected.

Neural noise was not assessed directly in this study, since no neuroimaging measures were included. Also, as previously discussed, whether differences in performance imply differences in the level of neural noise is unclear, since neural noise in older adults has been found to vary based on the neuroimaging technique or brain region under consideration. In a BOLD imaging study, Garrett and colleagues (2010) suggest that both lower and higher neural noise can reflect poorer brain function. The older adults showed lower noise than younger adults across the brain, which could have indicated less network complexity and greater losses in white matter, while older adults also showed greater noise than the younger adults in some brain regions, which could have indicated compensatory processes intended to maintain neural connections with aging. Thus, lower levels of noise in neural measures do not necessarily indicate better brain functioning or integrity of brain structures, and levels of neural noise cannot be reliably inferred from variability in behaviour. The lack of relationship between aging and increasing intraindividual variability observed in the present study, as reflected by no differences in the precision of motion estimates between the younger adults and older adult groups and the lack of correlation between age and lower precision across the older adults, could reflect multiple 
possibilities in terms of neural noise (e.g., neural noise did not increase with aging in our sample of older adults, or increases in neural noise with aging helped to create more resilient connections between neurons that support precision of motion perception).

It is most likely that the older adults did not show greater variability in their performance on the FGMI task because it involves first-order motion. Some researchers suggest that motion perception with aging may affect the perception of second-order motion more than first-order motion, due to the higher processing demands associated with the complexity of second-order motion (Faubert, 2002). The lower processing demands of the first-order motion involved in the FGMI task could have allowed the older adults to perform more consistently. The use of the FGMI task in this study was an important first step in addressing the question of whether differences in complexity between first-order and second-order motion are responsible for these findings of preserved precision of motion perception with aging, since the use of a first-order motion perception task did not introduce the additional complication of the higher stimulus complexity associated with second-order motion. The findings of the present study can now be contrasted with the findings of a future study using a second-order motion perception task. If precision is found to be poorer in older adults than younger adults only on the second-order motion perception task, it would be reasonable to infer that the higher complexity of second order motion perception could be responsible for this finding.

If loss of peripheral photoreceptors with aging are responsible for the greater FGMI exaggeration in peripheral vision in the older adults with poor stereopsis, one potential explanation for the finding of no significant difference in decreases in peripheral accuracy relative to central accuracy between younger adults and older adults with intact stereopsis is that these greater losses in peripheral photoreceptors only occurred in older adults with poor 
stereopsis. While the older adults with intact stereopsis did not have significantly larger decreases in their peripheral accuracy relative to their central accuracy than the younger adults, the median of the vision accuracy difference scores for the older adults with intact stereopsis was midway between the younger adults and the older adults with poor stereopsis, which could suggest a continuum of worsening peripheral accuracy. With less within-group variability in each of the three groups, which may have been achieved with a larger sample size, the older adults with intact stereopsis may have had larger decreases in their peripheral accuracy relative to their central accuracy than the younger adults.

\section{Relationships between Stereoacuity Losses in the Context of Aging and Central and Peripheral Accuracy and Precision of Motion Estimates}

The finding of higher central and peripheral vision accuracy of motion estimates (i.e., lower FGMI exaggeration) in younger adults and older adults with intact stereopsis than in older adults with poor stereopsis is congruent with previous research in which stereoacuity losses predicted greater FGMI exaggeration in individuals with strabismus (Reed \& Burdett, 2002). If, as some researchers suggest (Reed \& Burdett, 2002; Thompson \& Nawrot, 1999), stereopsis and motion perception share a neural mechanism, it is possible that the lower accuracy of the older adults with poor stereopsis, relative to the younger adults and older adults with intact stereopsis, is explained by a breakdown in a positional mechanism. The results of neuroimaging studies suggest the existence of a motion-processing system for first-order and second-order motion that involves regions of area MT, the dorsal intraparietal sulcus, and superior temporal sulcus (Claeys et al., 2003). There is also evidence of separate populations of neurons within area MT that respond to first-order and second-order motion (Ashida et al., 2007), and of large populations of neurons across the primary visual cortex that are sensitive to depth (DeAngelis \& Uka, 2003; 
Neri et al., 2004). Thus, the neuronal basis for the breakdown in this positional mechanism, as signaled by stereopsis losses, could be related to: (1) changes in the structure (e.g., cell death) or function (e.g., neurotransmitter levels) of the neuronal populations of area MT responsible for processing first-order motion, (2) changes in the structure or function of the neuronal populations that support stereopsis perception, or (3) the interaction of changes across these neuronal populations, particularly given the large number of neurons responsible for processing first-order motion that would also be sensitive to depth. However, the specific nature of these potential changes has not been established, since no literature has contrasted structural (e.g., brain volumes) and functional (e.g., BOLD) neural measures of older adults with intact stereopsis with those of older adults with poor stereopsis. In the primary visual cortices of rhesus monkeys, aging was associated with decreases in the integrity of myelin (Peters et al., 2000) and increases in the thickness of myelin (Peters et al., 2001), which could suggest structural changes to these populations of neurons. However, a review citing limited evidence of structural changes in visual cortex with normal aging concluded that functional changes are responsible for age-related declines in visual function (Andersen, 2012), which could suggest functional changes to these populations of neurons are more likely than structural changes.

It is also possible that the finding of lower accuracy in the older adults with poor stereopsis, relative to the younger adults and older adults with intact stereopsis, could suggest stereopsis losses signaled changes in oculomotor function in addition to or instead of changes in the neuron populations. Although eye position was not tracked in this study, changes in oculomotor function with aging, such as exophoria and esophoria, could have made it more difficult for some participants to ascertain the position of the first and second dots when making distance estimates. Tarita-Nistor and colleagues (2012) found that older adults with age-related 
macular degeneration showed more horizontal drift than younger adults during monocular fixation with the eye with worse visual acuity. Kosnik and colleagues (1986), while not finding any differences in fixation behaviour between younger and older adults at the group level, concluded that some of their older adults showed more deviation along the horizontal meridian that was likely unrelated to aging. These findings suggest that some older adults may show more horizontal drift during fixation, which could have contributed to greater FGMI exaggeration in the older adults with poor stereopsis, since the FGMI task involves the perception of motion in the horizontal plane.

Given the high prevalence of binocular and eye movement disorders in older adults (Leat et al., 2013), it is also possible that older adults with poor stereopsis might have other visual perception issues that contribute to FGMI exaggeration, such as visual confusion (the perception of two objects in the same space) or diplopia (the perception of one object in two locations). In the present study, visual confusion was unlikely, since participants reported the perception of a single dot moving. Diplopia, which is commonly reported in older adults (Leat et al., 2013) and can occur under monocular viewing conditions (Fincham, 1963; Woods, Bradley, \& Atchison, 1996), is a possible explanation, since it could have affected motion estimates by contributing perceptual noise that interfered with the ability to accurately determine the position of the first and second dots. Monocular diplopia typically occurs through the interaction of ocular spherical aberrations and defocus (Woods et al., 1996), a set of viewing conditions more likely to occur in older adults, since aging is associated with a range of visual declines (Anderson, 2012; Spear, 1993) including ocular spherical aberrations (e.g., astigmatism) and issues with focus (e.g., visual acuity losses). 
If changes in oculomotor function contributed to the lower accuracy of older adults with poor stereopsis, it would have been reasonable to expect that older adults with poor stereopsis would have also shown lower precision, as phoria and diplopia in the viewing eye might increase variability in older adults' motion estimates rather than consistently inflate their motion estimates. However, the results of previous research has shown eye drift in older adults is more common along the horizontal meridian than the vertical meridian (Kosnik et al., 1986; TaritaNistor et al., 2012), and exophoria may be more common than esophoria (Tarita-Nistor et al., 2012). Thus, it is possible that older adults with poor stereopsis generally showed exophoria in the viewing eye that increased the exaggeration of their FGMI estimates to a relatively consistent extent, which would not have increased variability. Given that older adults with poor stereopsis did not show lower precision than the younger adults and older adults with intact stereopsis, exophoria could be a more plausible explanation than diplopia for the finding of lower accuracy in older adults with poor stereopsis, since diplopia would be more likely to increase variability than to consistently inflate FGMI estimates. Diplopia can occur both horizontally and vertically (Woods et al., 1996). However, diplopia is found more often vertically than horizontally (Archer, 2007; Fincham, 1963), which makes diplopia less likely to consistently inflate FGMI estimates than the horizontal eye drift of exophoria, given that the FGMI movement occurs in the horizontal plane. More research is needed to determine the potential roles of exophoria and diplopia in FGMI perception, and it is not known whether participants in the present study showed either condition.

In the present study, as expected, FGMI exaggeration was found in both central and peripheral vision for all three groups. Although the motion estimates of the older adults with intact stereopsis did not quite reach significance for central vision, the median of the motion 
estimates for the older adults with intact stereopsis did exceed the actual 8-pixel ( $2 \mathrm{~mm})$ distance from the centre of the first dot to the centre of the second dot. The source of FGMI exaggeration is not fully understood, but it has been speculated that larger distances between photoreceptors and larger cortical receptive fields contribute to larger motion estimates (Foster et al., 1981; Reed $\&$ Burdett 2002). The degree of FGMI exaggeration for each group was greater in peripheral vision than in central vision, as reflected by the observation that the median accuracy values for each group were larger in peripheral vision than in central vision. This observation is likely representative of the increasing distance between photoreceptors and increases in cortical receptive field sizes as one moves from central to peripheral vision. It is also possible that exophoria in the covered eye could have contributed to the consistent finding of exaggerated motion perception in both central and peripheral vision for all three groups. In both younger adults with normal vision and older adults with age-related macular degeneration, Tarita-Nistor and colleagues (2012) found that a substantially larger percentage of participants showed exophoria than showed esophoria in the covered eye. Exophoria in the covered eye could have increased motion estimates for all three groups in central and peripheral vision by contributing perceptual noise that could interfere with the brain's ability to correctly interpret the corollary discharge associated with the movement of the viewing eye, possibly resulting in the perception of a larger movement of the viewing eye and an exaggeration of the motion estimate.

The results for the analyses examining the relationships between stereoacuity and central and peripheral precision were not as consistent as they were for aging. Taken together, the lack of a group effect on precision, and the lack of a correlation between stereoacuity and central precision across the older adults, suggest that stereoacuity losses in the context of aging had no relationship with the precision of motion estimates. However, in contrast, the correlation 
between lower stereoacuity and lower peripheral precision across the older adults could suggest stereoacuity losses in older adults are associated with precision deficits specific to peripheral vision. As discussed earlier, in reference to the lower accuracy of the older adults with poor stereopsis, relative to the younger adults and older adults with intact stereopsis, stereoacuity losses could have signaled structural or functional changes across neuronal populations responsive to first-order motion perception and depth. These changes could have resulted in relatively consistent changes to neuronal receptive fields, leading to consistent increases in FGMI exaggeration in the older adults with poor stereopsis. By this account, the combination of these changes across these neuronal populations with the greater loss of peripheral photoreceptors than central photoreceptors with aging (Gao \& Hollyfield, 1992), if they occurred to a greater extent in the older adults with poor stereopsis than in the older adults with intact stereopsis as previously suggested, could be the primary factor explaining the trend between lower stereoacuity and lower peripheral precision across the older adults, since sensory neurons would have less information to work with due to the larger distances between photoreceptors in peripheral vision than central vision. A trend between lower stereoacuity and lower central precision did not occur, possibly due to lower losses of central photoreceptors than peripheral photoreceptors with aging (Gao \& Hollyfield, 1992).

The trend toward larger decreases in peripheral accuracy relative to central accuracy in older adults with poor stereopsis than in younger adults was expected, given greater losses in peripheral photoreceptors than in central photoreceptors with aging (Gao \& Hollyfield, 1992). The greater loss of peripheral photoreceptors with aging would result in greater distances between photoreceptors in peripheral vision than in central vision, which could interact with other changes in oculomotor or neural function that are signaled by stereopsis losses, ultimately 
leading to greater FGMI exaggeration in peripheral vision than in central vision. However, the older adults with intact stereopsis, relative to the younger adults, did not also show larger decreases in peripheral accuracy relative to central accuracy. A potential explanation for these contrasting findings is that the older adults with poor stereopsis had greater losses in peripheral photoreceptors than central photoreceptors while the older adults with intact stereopsis did not. Thus, although age was not correlated with peripheral accuracy across the older adults, stereoacuity losses in the context of aging could have signaled greater deficits in vision with aging, which coincided with losses of peripheral photoreceptors.

The finding of no significant differences between the three groups on decreases in their peripheral accuracy relative to their central accuracy echoes the finding of no significant differences between the groups on central or peripheral precision, and similarly runs contrary to the predictions of the neural noise hypothesis and other theories related to increasing intraindividual variability with aging. As discussed in the context of the lower accuracy but similar precision of the older adults with poor stereopsis, relative to the younger adults and the older adults with intact stereopsis, a possible explanation for this finding is that stereopsis losses signal neural or oculomotor changes in the context of aging to inflate FGMI estimates to a relatively consistent degree in older adults with poor stereopsis.

\section{Limitations}

The present study had four important limitations. First, the relationships between advanced aging and central and peripheral accuracy and precision on the FGMI task were not examined. In the present study, the age range of the older adults extended from 66 to 89 years of age, but very few of the participants were over the age of 80 . The findings of the present study found no significant differences between younger adults and older adults with intact stereopsis in 
central or peripheral accuracy or precision, and limited evidence to suggest that having more subjects over the age of 80 would have changed the pattern of results for the correlations examining the relationship of age with central and peripheral accuracy and precision in older adults. The correlations of age with central precision and peripheral precision were small, not significant, and in the opposite direction, while the correlation of age with central accuracy showed no relationship and the correlation of age with peripheral accuracy was small, not significant, and in the opposite direction. However, while the extent to which individuals experience declines associated with aging depends on a range of environmental and biological factors (Brooks-Wilson, 2013; Lin et al., 2012), disorders of binocular vision and eye movement are more commonly found in advanced aging (Leat et al., 2013), which supports the speculation that advanced aging alone might be associated with subtle deficits even in the absence of these disorders. Thus, a group of oldest-old adults with intact stereopsis might show significantly lower central and peripheral accuracy and precision than younger adults, and correlations between age and lower central and peripheral accuracy and precision across the older adults. These relationships could also be stronger in oldest-old adults with poor stereopsis, since stereopsis losses in the present study were associated with lower central and peripheral accuracy.

Second, it remains to be established how age and stereoacuity losses would affect the performance of other motion perception tasks. The FGMI task involves the perception of finegrain, first-order movement, as the perception of motion is created by changes in luminance over a 2-mm distance from the centre of the first dot to the centre of the second dot. Thus, these findings may be specific to first-order motion, fine-grain movement, or to the task itself. The work of Claeys and colleagues (2003) suggest a single motion-processing system for first-order and second-order motion spanning regions of area MT, the dorsal intraparietal sulcus, and 
superior temporal sulcus. However, within area MT, separate populations of neurons may respond to first-order and second-order motion (Ashida et al., 2007). Thus, it is possible that age and stereoacuity losses would show similar or contrasting relationships with second-order motion, depending on whether aging and stereoacuity losses had similar or contrasting relationships with changes in these neuron populations.

Third, the present study was a behavioural study with no neuroimaging or eye tracking measures. As a result, it was only possible to use existing neuroimaging and eye tracking literature to speculate about the basis for the poorer vision accuracy of the estimates in older adults. The design of the present study did not allow for the determination of whether the lower central and peripheral accuracy observed in older adults with poor stereopsis, relative to the younger adults and older adults with intact stereopsis, was specifically due to stereopsis losses, neural changes, oculomotor changes, or an interaction of these changes. However, the behavioural results of this study, when placed in the context of the literature and the underlying logic of statistical testing, can support some inferences. The younger adults and older adults with intact stereopsis did not significantly differ in accuracy of their linear estimates, while older adults with poor stereopsis showed significantly lower central and peripheral accuracy than the younger adults and older adults with intact stereopsis. The lack of difference between the younger adults and older adults with intact stereopsis on accuracy, and the lack of correlation with age and central or peripheral accuracy across the older adults, suggests that age alone did not affect accuracy. Further, given that stereopsis losses were the primary factor that distinguished the two older adult groups, it is reasonable to infer that stereopsis losses signaled, or contributed to, changes in the context of aging that were responsible for the lower accuracy of the older adults with poor stereopsis. Attributing these findings of lower accuracy to 
breakdowns in a positional mechanism, due to neuronal or oculomotor changes that support motion perception, is a workable hypothesis for testing in future research.

Finally, it is possible that some effects were not detected due to the lack of power associated with a small sample size. Most of the effect sizes for the nonsignificant relationships were negligible, while the effect sizes associated with the significant and trending relationships were small or bordering on medium, which suggests sample size was likely not the issue for most of the nonsignificant relationships. However, the relationship between poorer stereoacuity and lower central precision might have been significant with a larger sample, given the effect size for this relationship was roughly halfway between small and moderate, not negligible. Neither older adult group showed more intraindividual variability (lower precision) than the younger adult group in central or peripheral vision at the group level. These findings ran contrary to the findings of previous literature and the predictions of the neural noise hypothesis and other theories regarding the nature of increasing intraindividual variability with aging. However, as discussed earlier, the most reasonable conclusion was that the findings suggest the FGMI task is not complex enough to be disrupted by increasing neural noise with aging. This interpretation of preserved precision in the context of aging alone is supported by the observations that the median of the precision values for the older adults with intact stereopsis were lower than in the younger adults for both central and peripheral vision, and that the correlation of age with central and peripheral precision across the older adults were small, not significant, and in the opposite direction.

\section{Future Directions}

Future studies could build on this study by addressing its limitations. First, the question of how advanced aging affects central and peripheral accuracy and precision could be addressed 
more directly by the inclusion of a group of oldest-old adults. While the findings of the present study indicated no significant effects of aging on central and peripheral accuracy and precision, it is possible that advanced aging may negatively affect these measures for the reasons discussed in the limitations section. Second, the question of how age and stereoacuity would affect the performance on other motion perception tasks could be addressed by substituting the FGMI task with other first-order motion perception tasks, or tasks defined by second-order motion or larger scale motion. Other questions that could be considered in future studies are whether these relationships change when the motion is vertical vs. horizontal or near vs. far. Finally, the present study was a behavioural study with no neuroimaging measures or eye tracking. While the behavioural data in the older adults with poor stereopsis suggest a potential deficit in a mechanism responsible for positional information, it is unclear whether the basis is neural or oculomotor. Neuroimaging and eye tracking data would allow for a more thorough comparison of the neural and oculomotor underpinnings of these behavioural outcomes in older adults with intact and poor stereopsis.

\section{Significance}

Motion perception plays an important role in supporting older adults' navigation abilities. However, findings in the literature are mixed as to whether aging negatively affects motion perception (Faubert, 2002; Spear, 1993). The results of the present study offer evidence to suggest that stereopsis losses contribute to findings of motion perception deficits in older adults. Older adults with poor stereopsis, relative to younger adults and older adults with intact stereopsis, showed a trend towards lower accuracy in their motion estimates for the FGMI task. In contrast, younger adults and older adults with intact stereopsis did not significantly differ in the accuracy of their motion estimates. The results of this study suggest that researchers would 
do well to consider the potential contributions of stereopsis losses to findings of motion perception deficits in older adults. By measuring stereopsis in older adults and examining the relationships of these measures with behavioural and neuroimaging measures, we should be able to arrive at a better sense of whether changes in motion perception are related to aging or to changes in stereoacuity. 


\section{Appendices}

\section{Appendix A: Visual and Medical History (Older Adults)}

1) How old are you? ___ yrs

2) Have you had your vision checked within the past two years? Yes No

3) What was the date of your last eye appointment?

4) Do you wear corrective lenses (e.g., glasses, contacts) or use any vision aids? Yes No If Yes: Would you be able to bring these to the study? Yes No

If Yes: What is your current prescription? OD: OS:

If NO: Without your corrective lenses, will you be able to read text from a page or read an eye chart clearly? Yes No

5) For this section, I want to know whether or not you have any of the medical conditions I am going to list to you. Please say "Yes" if you currently have this condition, or have had this condition in the past. And say "No" if you do not currently have this condition and have never had it in the past.

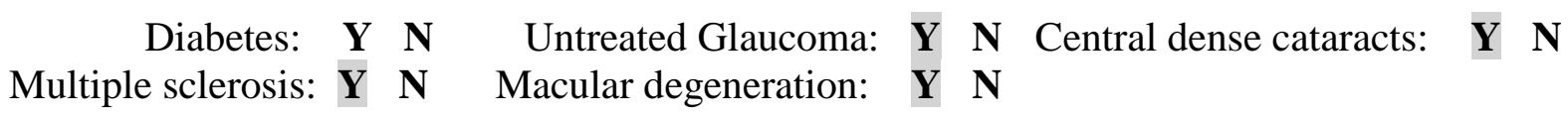

6) Do you have any other visual disorders or medical conditions which may affect vision? If so, could you list them?

7) For this section, I want to know whether or not you have had any of the following conditions clinically diagnosed by a professional (e.g., a doctor or a psychiatrist). Again, please say "Yes" if you currently have this condition, or have had this condition in the past. And say "No" if you do not currently have this condition and have never had it in the past.

Dementia: $\mathbf{Y} \quad$ Any other psychiatric disorders: $\mathbf{Y} \mathbf{N}$

Review the survey to make sure all of the questions have responses. If anything highlighted in gray has been circled, this person is not eligible to participate in the study. At the top of the page circle YES if eligible, and circle NO if ineligible. If the person is eligible, book them in. If the person is not eligible ... Unfortunately, you are not eligible for the study. However, you will still remain a member of the Ryerson Senior Participant Pool and may be contacted again in the future.

Thank you for your time. Have a nice day.

Eligible: YES NO

ID \# 


\section{Telephone Recruitment Script (Older Adults)}

“Hello, my name is Marlena Pearson. I am a Master's student in psychology at Ryerson University. I'm calling because you are part of the Ryerson Senior Participant Pool and we currently have a study you may be eligible to participate in. The purpose of this research is to assess whether age-related losses in depth perception affect people's ability to perceive motion. If you are eligible to participate in the study, we will ask you to come to the Ryerson University campus to complete several short tasks. In total, these tasks should take 1 to 1.5 hours to complete, and you will be given $\$ 15$ for your time. The tasks include a 10 minute cognitive assessment in which you will draw shapes, name pictures, and remember some short lists. Then we have three short vision tests, each should take approximately 5 minutes to complete. The first test is to assess depth perception, the second is to assess visual acuity (distance vision), and the third is to assess the extent you focus with an area of your eye other than your fovea. Finally, during the experimental task you will see a dot move across a computer screen, and be asked to report how far you thought the dot moved. We will be testing 102 people in total; would you like to participate?"

If the person says they are interested in participating: "That's great! Thank you. I have some extra inclusion and exclusion criteria for this study. Would you be willing to participate in a 5-minute survey now so I can make sure you are eligible?" If "YES": continue to Older Adult Visual Medical History

If the person says they are not interested in participating: "That's alright. Your decision not to participate in this study will not affect your relationship with Ryerson University. Would you like to be contacted for future studies?" (Appropriately update the Ryerson Senior Participant Pool depending on their response) "Thank you for your time. Have a nice day."

When administering the survey, ask all of the questions and record each response in the appropriate place. Regardless of the person's responses complete the survey (ask every question), then tell the person whether or not he or she is eligible.

\section{If the person is eligible:}

1. Book an appointment for him or her to come to the lab.

2. Let the person know where the study will take place at Ryerson University and where to meet you for their appointment. For example, "The study takes place on the Ryerson University campus, in the South Bond Building at 105 Bond St, in room 232. When you arrive, take the elevator or stairs to the second floor, and I will meet you in the second floor waiting area located directly in front of the elevator/stairs."

3. Leave your contact information with the participant in case they need to cancel or reschedule their appointment. For example, "I'm going to leave my contact information with you in case you need to reschedule or cancel this appointment. Again, my name is Marlena. You can reach me by telephone at the lab at (416) 979-5000 ext. 4681. If no one answers please leave a message, and I will return your call as soon as possible. Thank you again for your time. I will see you on (appointment date) at (appointment time). Have a great day!"

4. Appropriately update the Ryerson Senior Participant Pool.

\section{If the person is not eligible:}

"I'm very sorry, but it seems you are not eligible to participate in this study. However, if you are still interested we can keep your contact information in the Ryerson Senior Participant Pool and you will be contacted in the future. Thank you again for your time. Have a great day!" 


\section{Appendix B: Visual and Medical History (Younger Adults)}

1) How old are you? __ yrs

2) Have you had your vision checked within the past two years? Yes No

3) What was the date of your last eye appointment?

4) Do you wear corrective lenses (e.g., glasses, contacts)? Yes No

If Yes: What is your current prescription? OD: , OS:

5) Do you have any history of visual disorders (e.g., an eye that is crossed or poor visual acuity)?

Yes No

If Yes: what are they?

6) Do you have any medical conditions known to affect vision (e.g., diabetes)? Yes No If Yes: what are they?

At the end of the testing session, review the survey to make sure all of the questions have responses. If anything highlighted in gray has been circled, this person is not eligible to participate in the study. At the top of the page circle YES if eligible, and circle NO if ineligible.

Eligible: YES NO

ID \# 


\section{Appendix C: Montreal Cognitive Assessment (MoCA)}

MONTREAL COGNITIVE ASSESSMENT (MOCA)

ID \#

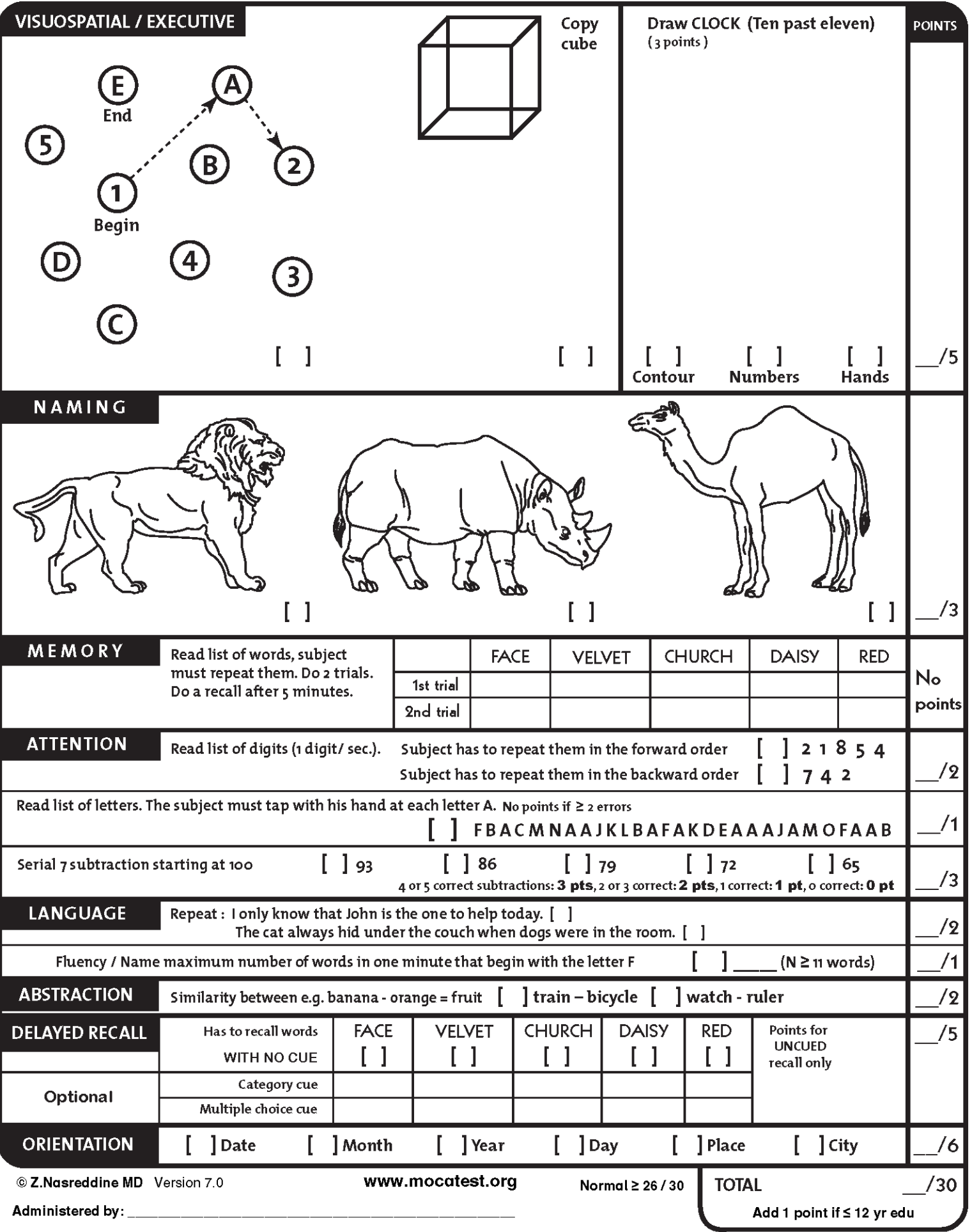




\section{MoCA Adminstration and Scoring Instructions}

The Montreal Cognitive Assessment (MoCA) was designed as a rapid screening instrument for mild cognitive dysfunction. It assesses different cognitive domains: attention and concentration, executive functions, memory, language, visuoconstructional skills, conceptual thinking, calculations, and orientation. Time to administer the MoCA is approximately 10 minutes. The total possible score is 30 points; a score of 26 or above is considered normal.

1. Alternating Trail Making:

Administration: The examiner instructs the subject: "Please draw a line, going from a number to a letter in ascending order. Begin here [point to (1)] and draw a line from 1 then to A then to 2 and so on. End here [point to $(\mathrm{E})] . "$

Scoring: Allocate one point if the subject successfully draws the following pattern: 1- A- 2- B- 3- C- 4- D- 5- E, without drawing any lines that cross. Any error that is not immediately self-corrected earns a score of 0 .

2. Visuoconstructional Skills (Cube):

Administration: The examiner gives the following instructions, pointing to the cube: "Copy this drawing as accurately as you can, in the space below".

Scoring: One point is allocated for a correctly executed drawing.

- $\quad$ Drawing must be three-dimensional

- $\quad$ All lines are drawn

- $\quad$ No line is added

- $\quad$ Lines are relatively parallel and their length is similar (rectangular prisms are accepted)

A point is not assigned if any of the above-criteria are not met.

3. Visuoconstructional Skills (Clock):

Administration: Indicate the right third of the space and give the following instructions:

"Draw a clock. Put in all the numbers and set the time to 10 after 11 ".

Scoring: One point is allocated for each of the following three criteria:

- $\quad$ Contour (1 pt.): the clock face must be a circle with only minor distortion acceptable (e.g., slight imperfection on closing the circle);

- $\quad$ Numbers (1 pt.): all clock numbers must be present with no additional numbers; numbers must be in the correct order and placed in the approximate quadrants on the clock face; Roman numerals are acceptable; numbers can be placed outside the circle contour;

- $\quad$ Hands (1 pt.): there must be two hands jointly indicating the correct time; the hour hand must be clearly shorter than the minute hand; hands must be centred within the clock face with their junction close to the clock centre.

A point is not assigned for a given element if any of the above-criteria are not met.

4. Naming:

Administration: Beginning on the left, point to each figure and say: "Tell me the name of this animal". 
Scoring: One point each is given for the following responses: (1) camel or dromedary, (2) lion, (3) rhinoceros or rhino.

\section{Memory:}

Administration: The examiner reads a list of 5 words at a rate of one per second, giving the following instructions: "This is a memory test. I am going to read a list of words that you will have to remember now and later on. Listen carefully. When I am through, tell me as many words as you can remember. It doesn't matter in what order you say them". Mark a check in the allocated space for each word the subject produces on this first trial. When the subject indicates that (s)he has finished (has recalled all words), or can recall no more words, read the list a second time with the following instructions: "I am going to read the same list for a second time. Try to remember and tell me as many words as you can, including words you said the first time." Put a check in the allocated space for each word the subject recalls after the second trial.

At the end of the second trial, inform the subject that (s)he will be asked to recall these words again by saying, "I will ask you to recall those words again at the end of the test."

Scoring: No points are given for Trials One and Two.

\section{Attention:}

Forward Digit Span: Administration: Give the following instruction: "I am going to say some numbers and when I am through, repeat them to me exactly as I said them". Read the five number sequence at a rate of one digit per second.

Backward Digit Span: Administration: Give the following instruction: "Now I am going to say some more numbers, but when I am through you must repeat them to me in the backwards order." Read the three number sequence at a rate of one digit per second.

Scoring: Allocate one point for each sequence correctly repeated, (N.B.: the correct response for the backwards trial is 2-4-7).

Vigilance: Administration: The examiner reads the list of letters at a rate of one per second, after giving the following instruction: "I am going to read a sequence of letters. Every time I say the letter A, tap your hand once. If I say a different letter, do not tap your hand".

Scoring: Give one point if there is zero to one errors (an error is a tap on a wrong letter or a failure to tap on letter A).

Serial 7s: Administration: The examiner gives the following instruction: "Now, I will ask you to count by subtracting seven from 100, and then, keep subtracting seven from your answer until I tell you to stop." Give this instruction twice if necessary.

Scoring: This item is scored out of 3 points. Give no (0) points for no correct subtractions, 1 point for one correction subtraction, 2 points for two-to-three correct subtractions, and 3 points if 
the participant successfully makes four or five correct subtractions. Count each correct subtraction of 7 beginning at 100. Each subtraction is evaluated independently; that is, if the participant responds with an incorrect number but continues to correctly subtract 7 from it, give a point for each correct subtraction. For example, a participant may respond "92-85-78-7164 " where the "92" is incorrect, but all subsequent numbers are subtracted correctly. This is one error and the item would be given a score of 3 .

\section{Sentence repetition:}

Administration: The examiner gives the following instructions: "I am going to read you a sentence. Repeat it after me, exactly as I say it [pause]: I only know that John is the one to help today." Following the response, say: "Now I am going to read you another sentence. Repeat it after me, exactly as I say it [pause]: The cat always hid under the couch when dogs were in the room."

Scoring: Allocate 1 point for each sentence correctly repeated. Repetition must be exact. Be alert for errors that are omissions (e.g., omitting "only", "always") and substitutions/additions (e.g., "John is the one who helped today;" substituting "hides" for "hid", altering plurals, etc.).

\section{Verbal fluency:}

Administration: The examiner gives the following instruction: "Tell me as many words as you can think of that begin with a certain letter of the alphabet that I will tell you in a moment. You can say any kind of word you want, except for proper nouns (like Bob or Boston), numbers, or words that begin with the same sound but have a different suffix, for example, love, lover, loving. I will tell you to stop after one minute. Are you ready? [Pause] Now, tell me as many words as you can think of that begin with the letter F. [time for $60 \mathrm{sec}$. Stop."

Scoring: Allocate one point if the subject generates 11 words or more in $60 \mathrm{sec}$. Record the subject's response in the bottom or side margins.

\section{Abstraction:}

Administration: The examiner asks the subject to explain what each pair of words has in common, starting with the example: "Tell me how an orange and a banana are alike". If the subject answers in a concrete manner, then say only one additional time: "Tell me another way in which those items are alike". If the subject does not give the appropriate response (fruit), say, "Yes, and they are also both fruit." Do not give any additional instructions or clarification. After the practice trial, say: "Now, tell me how a train and a bicycle are alike". Following the response, administer the second trial, saying: "Now tell me how a ruler and a watch are alike". Do not give any additional instructions or prompts.

Scoring: Only the last two item pairs are scored. Give 1 point to each item pair correctly answered. The following responses are acceptable:

Train-bicycle $=$ means of transportation, means of travelling, you take trips in both; Ruler-watch $=$ measuring instruments, used to measure. 
The following responses are not acceptable: Train-bicycle $=$ they have wheels; Ruler-watch $=$ they have numbers.

10. Delayed recall:

Administration: The examiner gives the following instruction: "I read some words to you earlier, which I asked you to remember. Tell me as many of those words as you can remember. Make a check mark for each of the words correctly recalled spontaneously without any cues, in the allocated space.

Scoring: Allocate 1 point for each word recalled freely without any cues.

Optional:

Following the delayed free recall trial, prompt the subject with the semantic category cue provided below for any word not recalled. Make a check mark in the allocated space if the subject remembered the word with the help of a category or multiple-choice cue. Prompt all nonrecalled words in this manner. If the subject does not recall the word after the category cue, give $\mathrm{him} /$ her a multiple choice trial, using the following example instruction, "Which of the following words do you think it was, NOSE, FACE, or HAND?"

Use the following category and/or multiple-choice cues for each word, when appropriate: FACE: category cue: part of the bodymultiple choice: nose, face, hand VELVET: category cue:

type of fabric multiple choice: denim, cotton, velvet $\mathrm{CHURCH}$ : category cue: type of building multiple choice: church, school, hospital DAISY: category cue: type of flower multiple choice: rose, daisy, tulip

RED: category cue: a colour multiple choice: red, blue, green

Scoring: No points are allocated for words recalled with a cue. A cue is used for clinical information purposes only and can give the test interpreter additional information about the type of memory disorder. For memory deficits due to retrieval failures, performance can be improved with a cue. For memory deficits due to encoding failures, performance does not improve with a cue.

11. Orientation:

Administration: The examiner gives the following instructions: "Tell me the date today". If the subject does not give a complete answer, then prompt accordingly by saying: "Tell me the [year, month, exact date, and day of the week]." Then say: "Now, tell me the name of this place, and which city it is in."

Scoring: Give one point for each item correctly answered. The subject must tell the exact date and the exact place (name of hospital, clinic, office). No points are allocated if subject makes an error of one day for the day and date.

TOTAL SCORE: Sum all subscores listed on the right-hand side. Add one point for an individual who has 12 years or fewer of formal education, for a possible maximum of 30 points. A final total score of 26 and above is considered normal. 


\section{Appendix D: Statistical Summaries}

\section{Demographics}

\begin{tabular}{lccc}
\hline & $\begin{array}{c}\text { Younger } \\
\text { adults }\end{array}$ & $\begin{array}{c}\text { Older adults with } \\
\text { intact stereopsis } \\
(\mathrm{n}=12)\end{array}$ & $\begin{array}{c}\text { Older adults with } \\
\text { poor stereopsis } \\
(\mathrm{n}=10)\end{array}$ \\
\hline Age: $M d(M A D)$ & $18.00(0)$ & $73.00(4.00)$ & $70.00(3.50)$ \\
MoCA score: $\bar{x}(s)$ & $\mathrm{NA}$ & $26.75(2.42)$ & $27.20(1.62)$ \\
Corrected visual acuity: $M d(M A D)$ & $20 / 16(3.50)^{\mathrm{a}}$ & $20 / 20(0)^{\mathrm{b}}$ & $20 / 20(0)^{\mathrm{b}}$ \\
Stereoacuity: $M d(M A D)$ & $40.00(0)$ & $40.00(0)$ & $90.00(10.00)$ \\
\hline
\end{tabular}

For each variable, groups with different letters were significantly different from each other at $p<.05$.

Median absolute deviation $(M A D)=\operatorname{median}\left[\mid X_{i}-\operatorname{median}\left(X_{i} \mid\right)\right]$.

\section{Age comparisons between the two older adult groups}

\begin{tabular}{|ll|r|r|r|r|r|r|}
\hline \multicolumn{1}{|c|}{ Tests of Normality } \\
\cline { 3 - 8 } & \multicolumn{3}{|c|}{ Kolmogorov-Smirnova } & \multicolumn{3}{c|}{ Shapiro-Wilk } \\
\cline { 2 - 8 } & Group & Statistic & \multicolumn{1}{c|}{ Df } & \multicolumn{1}{c|}{ Sig. } & \multicolumn{1}{c|}{ Statistic } & df & \multicolumn{1}{c|}{ Sig. } \\
\hline Age & Older Intact Stereo & .185 & 12 & $.200^{\circ}$ & .922 & 12 & .299 \\
& Older Poor Stereo & .296 & 10 & .013 & .844 & 10 & .049 \\
\hline
\end{tabular}

a. Lilliefors Significance Correction

${ }^{*}$. This is a lower bound of the true significance.

Test of Homogeneity of Variance

\begin{tabular}{|ll|r|r|r|r|}
\hline & Levene Statistic & df1 & \multicolumn{1}{c|}{$\mathrm{df2}$} & \multicolumn{1}{c|}{ Sig. } \\
\hline Age & 7.507 & 1 & 20 & .013 \\
& Based on Mean & 1.418 & 1 & 20 & .248 \\
Based on Median & 1.418 & 1 & 11.752 & .257 \\
Based on Median and with & & & & \\
adjusted df & 6.720 & 1 & 20 & .017 \\
Based on trimmed mean & &
\end{tabular}

Test Statistics ${ }^{b}$

\begin{tabular}{|l|r|}
\hline & \multicolumn{1}{|c|}{ Age } \\
\hline Mann-Whitney U & 58.000 \\
Wilcoxon W & 113.000 \\
Z & -.133 \\
Asymp. Sig. (2-tailed) & .895 \\
Exact Sig. [2*(1-tailed Sig.)] & $.923^{a}$ \\
\hline
\end{tabular}

a. Not corrected for ties.

b. Grouping Variable: Group 


\section{MoCA comparisons between the two older adult groups.}

\begin{tabular}{|c|c|c|c|c|c|c|c|}
\hline & \multirow[b]{2}{*}{ Group } & \multicolumn{3}{|c|}{ Kolmogorov-Smirnova } & \multicolumn{3}{|c|}{ Shapiro-Wilk } \\
\hline & & Statistic & df & Sig. & Statistic & df & Sig. \\
\hline \multirow[t]{2}{*}{ MoCA Score } & Older Intact Stereo & .211 & 12 & .144 & .902 & 12 & .166 \\
\hline & Older Poor Stereo & .189 & 10 & $.200^{*}$ & .873 & 10 & .109 \\
\hline
\end{tabular}

a. Lilliefors Significance Correction

*. This is a lower bound of the true significance.

\section{Test of Homogeneity of Variance}

\begin{tabular}{|ll|r|r|r|r|}
\hline & Levene Statistic & df1 & df2 & Sig. \\
\hline MoCA Score & Based on Mean & .222 & 1 & 20 & .642 \\
& Based on Median & .092 & 1 & 20 & .765 \\
& Based on Median and with & .092 & 1 & 13.968 & .766 \\
& adjusted df & & & .709 \\
\hline
\end{tabular}

Independent Samples Test

\begin{tabular}{|c|c|c|c|c|c|c|c|c|c|c|}
\hline & \multicolumn{2}{|c|}{$\begin{array}{c}\text { Levene's Test for } \\
\text { Equality of } \\
\text { Variances }\end{array}$} & \multicolumn{7}{|c|}{ t-test for Equality of Means } \\
\hline & & \multirow[b]{2}{*}{$\mathrm{F}$} & \multirow[b]{2}{*}{ Sig. } & \multirow[b]{2}{*}{$\mathrm{T}$} & \multirow[b]{2}{*}{ df } & \multirow{2}{*}{$\begin{array}{l}\text { Sig. (2- } \\
\text { tailed) }\end{array}$} & \multirow{2}{*}{$\begin{array}{c}\text { Mean } \\
\text { Difference }\end{array}$} & \multirow{2}{*}{$\begin{array}{l}\text { Std. Error } \\
\text { Difference }\end{array}$} & \multicolumn{2}{|c|}{$\begin{array}{l}95 \% \text { Confidence } \\
\text { Interval of the } \\
\text { Difference }\end{array}$} \\
\hline & & & & & & & & & Lower & Upper \\
\hline $\begin{array}{l}\text { MoCA } \\
\text { Score }\end{array}$ & $\begin{array}{l}\text { Equal } \\
\text { variances } \\
\text { assumed } \\
\text { Equal } \\
\text { variances not } \\
\text { assumed }\end{array}$ & .222 & .642 & $\begin{array}{l}-.501 \\
-.520\end{array}$ & 20 & .622 & -.450 & .865 & -2.322 & 1.360 \\
\hline
\end{tabular}

Visual acuity comparisons amongst young adults, older adults with intact stereopsis, and older adults with poor stereopsis

\begin{tabular}{|c|c|c|c|c|c|c|c|}
\hline & \multirow[b]{2}{*}{ Group } & \multicolumn{3}{|c|}{ Kolmogorov-Smirnova } & \multicolumn{3}{|c|}{ Shapiro-Wilk } \\
\hline & & Statistic & $d f$ & Sig. & Statistic & $d f$ & Sig. \\
\hline \multirow[t]{2}{*}{$\begin{array}{l}\text { Corrected Visual Acuity } \\
\text { (Snellen Score) }\end{array}$} & $\begin{array}{l}\text { Younger Intact } \\
\text { Stereo }\end{array}$ & .202 & 34 & .001 & .843 & 34 & .000 \\
\hline & Older Poor Stereo & .352 & 10 & .001 & .748 & 10 & .003 \\
\hline
\end{tabular}

a. Lilliefors Significance Correction

b. Corrected Visual Acuity (Snellen Score) is constant when Group = Older Intact Stereo. It has been omitted. 
Test of Homogeneity of Variance ${ }^{a}$

\begin{tabular}{|ll|r|r|r|r|}
\hline & & Levene Statistic & df1 & df2 & \multicolumn{1}{c|}{ Sig. } \\
\hline Corrected Visual Acuity & Based on Mean & 1.711 & 1 & 42 & .198 \\
(Snellen Score) & Based on Median & 2.295 & 1 & 42 & .137 \\
& Based on Median and with & 2.295 & .137 \\
& adjusted df & 1 & 41.808 & \\
& Based on trimmed mean & & & .224 \\
\hline
\end{tabular}

a. Corrected Visual Acuity (Snellen Score) is constant when Group = Older Intact Stereo. It has been omitted.

Test Statistics ${ }^{\mathrm{a}, \mathrm{b}}$

\begin{tabular}{|l|r|}
\hline & \multicolumn{1}{|c|}{$\begin{array}{c}\text { Corrected } \\
\text { Visual Acuity } \\
\text { (Snellen Score) }\end{array}$} \\
\hline Chi-Square & 20.568 \\
df & 2 \\
Asymp. Sig. & .000 \\
\hline
\end{tabular}

a. Kruskal Wallis Test

b. Grouping Variable: Group

Visual acuity comparison between younger adults and older adults with intact stereopsis

\begin{tabular}{|c|c|}
\hline & $\begin{array}{c}\text { Corrected } \\
\text { Visual Acuity } \\
\text { (Snellen Score) }\end{array}$ \\
\hline $\begin{array}{l}\text { Mann-Whitney U } \\
\text { Wilcoxon W } \\
\text { Z } \\
\text { Asymp. Sig. (2-tailed) }\end{array}$ & $\begin{array}{r}60.000 \\
655.000 \\
-3.889 \\
.000\end{array}$ \\
\hline
\end{tabular}

a. Grouping Variable: Group

Visual acuity comparison between younger adults and older adults with poor stereopsis

\begin{tabular}{|l|r|}
\hline \multicolumn{2}{|c|}{ Test Statistics $^{\mathbf{b}}$} \\
\hline & $\begin{array}{c}\text { Corrected } \\
\text { Visual Acuity } \\
\text { (Snellen Score) }\end{array}$ \\
\hline Mann-Whitney U & 68.000 \\
Wilcoxon W & 663.000 \\
Z & -3.026 \\
Asymp. Sig. (2-tailed) & .002 \\
Exact Sig. [2*(1-tailed Sig.)] & $.003^{\mathrm{a}}$ \\
\hline
\end{tabular}

a. Not corrected for ties.

b. Grouping Variable: Group 


\section{Visual acuity comparison between the two older adult groups}

\begin{tabular}{|l|r|}
\hline \multicolumn{2}{|c|}{ Test Statistics $^{\mathbf{b}}$} \\
& \multicolumn{1}{|c|}{$\begin{array}{c}\text { Corrected } \\
\text { Visual Acuity } \\
\text { (Snellen Score) }\end{array}$} \\
\hline Mann-Whitney U & 54.000 \\
Wilcoxon W & 109.000 \\
Z & -.663 \\
Asymp. Sig. (2-tailed) & .507 \\
Exact Sig. [2*(1-tailed Sig.)] & $.722^{\mathrm{a}}$ \\
\hline
\end{tabular}

a. Not corrected for ties.

b. Grouping Variable: Group

Descriptive statistics for level of vision correction amongst the three groups

Descriptive Statistics

\begin{tabular}{|ll|r|r|r|r|r|r|}
\hline Group & & $\mathrm{N}$ & \multicolumn{1}{c|}{ Range } & Minimum & Maximum & Mean & Std. Deviation \\
\hline \multirow{2}{*}{ Younger Intact } & Rx OD & 9 & 2.00 & -2.00 & .00 & -.8889 & .67443 \\
Stereo & Rx OS & 9 & 1.25 & -1.75 & -.50 & -1.0278 & .53684 \\
& Valid N (listwise) & 9 & & & & & \\
\hline \multirow{2}{*}{ Older Intact Stereo } & Rx OD & 6 & 2.00 & -2.50 & -.50 & -1.0000 & .79057 \\
& Rx OS & 6 & 2.00 & -2.50 & -.50 & -1.2500 & .67082 \\
& Valid N (listwise) & 6 & & & & & \\
\hline \multirow{2}{*}{ Older Poor Stereo } & Rx OD & 10 & 2.00 & -2.50 & -.50 & -1.2000 & .80623 \\
& Rx OS & 10 & 2.50 & -2.50 & .00 & -.9500 & .71492 \\
& Valid N (listwise) & 10 & & & & & \\
\hline
\end{tabular}

Stereoacuity comparisons between younger adults and older adults with intact stereopsis

\begin{tabular}{|c|c|c|c|c|c|c|c|}
\hline & \multirow[b]{2}{*}{ Group } & \multicolumn{3}{|c|}{ Kolmogorov-Smirnov ${ }^{a}$} & \multicolumn{3}{|c|}{ Shapiro-Wilk } \\
\hline & & Statistic & $\mathrm{df}$ & Sig. & Statistic & df & Sig. \\
\hline \multirow[t]{2}{*}{ Stereoacuity (degrees) } & $\begin{array}{l}\text { Younger Intact } \\
\text { Stereo }\end{array}$ & .538 & 34 & .000 & .255 & 34 & .000 \\
\hline & Older Intact Stereo & .460 & 12 & .000 & .552 & 12 & .000 \\
\hline
\end{tabular}

a. Lilliefors Significance Correction

Test of Homogeneity of Variance

\begin{tabular}{|ll|r|r|r|r|}
\hline & & Levene Statistic & df1 & df2 & Sig. \\
\hline Stereoacuity (degrees) & Based on Mean & 13.440 & 1 & 44 & .001 \\
& Based on Median & 3.452 & 1 & 44 & .070 \\
& Based on Median and with & 3.452 & .073 \\
& adjusted df & & 30.085 & \\
& Based on trimmed mean & 13.440 & & 1 & .001 \\
\hline
\end{tabular}




\section{Test Statistics ${ }^{a}$}

\begin{tabular}{|l|r|}
\hline & $\begin{array}{c}\text { Stereoacuity } \\
\text { (degrees) }\end{array}$ \\
\hline Mann-Whitney U & 165.000 \\
Wilcoxon W & 760.000 \\
Z & -1.809 \\
Asymp. Sig. (2-tailed) & .070 \\
\hline
\end{tabular}

a. Grouping Variable: Group

\section{Normality test for FGMI exaggeration within each group for central vision accuracy}

Tests of Normality

\begin{tabular}{|c|c|c|c|c|c|c|c|}
\hline & \multirow[b]{2}{*}{ Group } & \multicolumn{3}{|c|}{ Kolmogorov-Smirnova } & \multicolumn{3}{|c|}{ Shapiro-Wilk } \\
\hline & & Statistic & $d f$ & Sig. & Statistic & $d f$ & Sig. \\
\hline \multirow[t]{3}{*}{ Central Accuracy } & Younger Intact Stereo & .132 & 34 & .139 & .942 & 34 & .072 \\
\hline & Older Intact Stereo & .224 & 11 & .130 & .857 & 11 & .053 \\
\hline & Older Poor Stereo & .125 & 10 & $.200^{*}$ & .955 & 10 & .722 \\
\hline
\end{tabular}

a. Lilliefors Significance Correction

*. This is a lower bound of the true significance.

\section{One-sample t-test of FGMI exaggeration for central vision accuracy for younger adults}

One-Sample Statistics

\begin{tabular}{|l|r|r|r|r|}
\hline & $\mathrm{N}$ & Mean & Std. Deviation & Std. Error Mean \\
\hline Younger_Central_Accuracy & 34 & 18.5026 & 9.78756 & 1.67855 \\
\hline
\end{tabular}

One-Sample Test

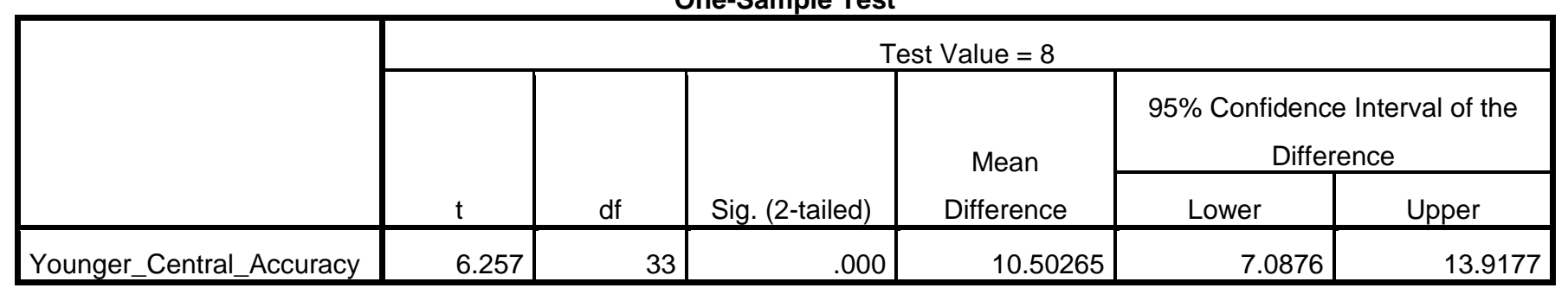


One-sample t-test of FGMI exaggeration for central vision accuracy for older adults with intact stereopsis

\begin{tabular}{|c|c|c|c|c|}
\hline \multicolumn{5}{|c|}{ One-Sample Statistics } \\
\hline & $\mathrm{N}$ & Mean & Std. Deviation & Std. Error Mean \\
\hline Older_Intact_Central_Accuracy & 11 & 17.5900 & 14.51321 & 4.37590 \\
\hline
\end{tabular}

One-Sample Test

\begin{tabular}{|c|c|c|c|c|c|c|}
\hline & \multicolumn{6}{|c|}{ Test Value $=8$} \\
\hline & \multirow[b]{2}{*}{$\mathrm{t}$} & \multirow[b]{2}{*}{ df } & \multirow{2}{*}{$\begin{array}{l}\text { Sig. (2- } \\
\text { tailed) }\end{array}$} & \multirow{2}{*}{$\begin{array}{c}\text { Mean } \\
\text { Difference } \\
\end{array}$} & \multicolumn{2}{|c|}{$\begin{array}{l}\text { 95\% Confidence Interval of the } \\
\text { Difference }\end{array}$} \\
\hline & & & & & Lower & Upper \\
\hline Older_Intact_Central_Accuracy & 2.192 & 10 & .053 & 9.59000 & -.1601 & 19.3401 \\
\hline
\end{tabular}

One-sample t-test of FGMI exaggeration for central vision accuracy for older adults with poor stereopsis

\begin{tabular}{|c|c|c|c|c|}
\hline \multicolumn{5}{|c|}{ One-Sample Statistics } \\
\hline & $\mathrm{N}$ & Mean & Std. Deviation & Std. Error Mean \\
\hline Older_Poor_Central_Accruacy & 10 & 35.0590 & 21.63610 & 6.84194 \\
\hline
\end{tabular}

One-Sample Test

\begin{tabular}{|c|c|c|c|c|c|c|}
\hline \multicolumn{7}{|c|}{ Test Value $=8$} \\
\hline & \multirow[b]{2}{*}{$\mathrm{t}$} & \multirow[b]{2}{*}{$d f$} & \multirow{2}{*}{$\begin{array}{l}\text { Sig. }(2- \\
\text { tailed) }\end{array}$} & \multirow{2}{*}{$\begin{array}{c}\text { Mean } \\
\text { Difference } \\
\end{array}$} & \multicolumn{2}{|c|}{$\begin{array}{l}\text { 95\% Confidence Interval of the } \\
\text { Difference }\end{array}$} \\
\hline & & & & & Lower & Upper \\
\hline Older_Poor_Central_Accruacy & 3.955 & 9 & .003 & 27.05900 & 11.5815 & 42.5365 \\
\hline
\end{tabular}


Central vison accuracy comparison amongst younger adults, older adults with intact stereopsis, and older adults with poor stereopsis

Test of Homogeneity of Variance

\begin{tabular}{|ll|r|r|r|r|}
\hline & \multicolumn{1}{|c|}{$\begin{array}{l}\text { Levene } \\
\text { Statistic }\end{array}$} & \multicolumn{1}{c|}{$\mathrm{df1}$} & \multicolumn{1}{c|}{$\mathrm{df2}$} & \multicolumn{1}{c|}{ Sig. } \\
\hline Central Accuracy & Based on Mean & 7.580 & 2 & 52 & .001 \\
& Based on Median & 5.149 & 2 & 52 & .009 \\
& Based on Median and with & 5.149 & 2 & 31.321 & .012 \\
& & & & \\
& adjusted df & & & \\
& Based on trimmed mean & 7.395 & 2 & .001 \\
\hline
\end{tabular}

Central Accuracy

\begin{tabular}{|l|r|r|}
\hline Group & N & Median \\
\hline Younger Intact Stereo & 34 & 17.3125 \\
Older Intact Stereo & 11 & 10.8750 \\
Older Poor Stereo & 10 & 33.4375 \\
Total & 55 & 17.5000 \\
\hline
\end{tabular}

Test Statistics ${ }^{a, b}$

\begin{tabular}{|l|r|}
\hline & \multicolumn{1}{|c|}{$\begin{array}{c}\text { Central } \\
\text { Accuracy }\end{array}$} \\
\hline Chi-Square & 5.446 \\
df & 2 \\
Asymp. Sig. & .066 \\
\hline
\end{tabular}

a. Kruskal Wallis Test

b. Grouping Variable: Group

Central vision accuracy comparison between younger adults and older adults with intact stereopsis

\begin{tabular}{|l|r|}
\hline \multicolumn{2}{|c|}{ Test Statistics $^{\mathbf{b}}$} \\
\hline Mann-Whitney U & \multicolumn{1}{|c|}{$\begin{array}{c}\text { Central } \\
\text { Accuracy }\end{array}$} \\
Wilcoxon W & 164.500 \\
Z & 230.500 \\
Asymp. Sig. (2-tailed) & -.594 \\
Exact Sig. [2*(1-tailed Sig.)] & .552 \\
\hline
\end{tabular}

a. Not corrected for ties.

b. Grouping Variable: Group 
Central vision accuracy comparison between younger adults and older adults with poor stereopsis

\begin{tabular}{|c|c|}
\hline & $\begin{array}{c}\text { Central } \\
\text { Accuracy }\end{array}$ \\
\hline $\begin{array}{l}\text { Mann-Whitney U } \\
\text { Wilcoxon W } \\
\text { Z } \\
\text { Asymp. Sig. (2-tailed) } \\
\text { Exact Sig. [2*(1-tailed Sig.)] }\end{array}$ & $\begin{array}{r}94.000 \\
689.000 \\
-2.129 \\
.033 \\
.033^{a}\end{array}$ \\
\hline
\end{tabular}

a. Not corrected for ties.

b. Grouping Variable: Group

Central vision accuracy comparison between the two older adult groups

\begin{tabular}{|l|r|}
\hline \multicolumn{2}{|c|}{ Test Statistics $^{\mathbf{b}}$} \\
\hline Mann-Whitney U & $\begin{array}{c}\text { Central } \\
\text { Accuracy }\end{array}$ \\
Wilcoxon W & 27.000 \\
Z & 93.000 \\
Asymp. Sig. (2-tailed) & -1.972 \\
Exact Sig. [2 & (1-tailed Sig.)] \\
\hline
\end{tabular}

a. Not corrected for ties.

b. Grouping Variable: Group

Normality test of FGMI exaggeration within each group for peripheral vision accuracy

\begin{tabular}{|ll|r|r|r|r|r|r|}
\hline & \multicolumn{1}{|c|}{ Tests of Normality } \\
\cline { 2 - 8 } & \multicolumn{2}{|c|}{ Kolmogorov-Smirnov } & \multicolumn{3}{|c|}{ Shapiro-Wilk } \\
\cline { 2 - 8 } & Group & \multicolumn{1}{c|}{ Statistic } & df & \multicolumn{1}{c|}{ Sig. } & Statistic & df & \multicolumn{1}{c|}{ Sig. } \\
\hline Peripheral Accuracy & Younger Intact Stereo & .159 & .030 & .932 & 34 & .035 \\
& Older Intact Stereo & .142 & 12 & $.200^{*}$ & .919 & 12 & .281 \\
& Older Poor Stereo & .267 & 10 & .042 & .770 & 10 & .006 \\
\hline
\end{tabular}

a. Lilliefors Significance Correction

*. This is a lower bound of the true significance.

One-sample Wilcoxon signed rank test of FGMI exaggeration for central vision accuracy

for younger adults

Hypothesis Test Summary

\begin{tabular}{|c|c|c|c|c|}
\hline & Null Hypothesis & Test & Sig. & Decision \\
\hline 1 & $\begin{array}{l}\text { The median of } \\
\text { Younger_Peripheral_Acourac } \\
\text { equals } 8.00 \text {. }\end{array}$ & $\begin{array}{l}\text { One-Sample } \\
\text { owilcoxon Signed } \\
\text { Rank Test }\end{array}$ & .000 & $\begin{array}{l}\text { Reject the } \\
\text { null } \\
\text { hypothesis. }\end{array}$ \\
\hline
\end{tabular}

Asymptotio signifioanoes are displayed. The signifioanoe level is, 05 . 
One-sample t-test of FGMI exaggeration for central vision accuracy for younger adults

One-Sample Statistics

\begin{tabular}{|l|r|r|r|r|}
\hline & N & Mean & Std. Deviation & Std. Error Mean \\
\hline $\begin{array}{l}\text { Older_Intact_Peripheral_Acc } \\
\text { uracy }\end{array}$ & 12 & 35.2825 & 19.16118 & 5.53136 \\
\hline
\end{tabular}

One-Sample Test

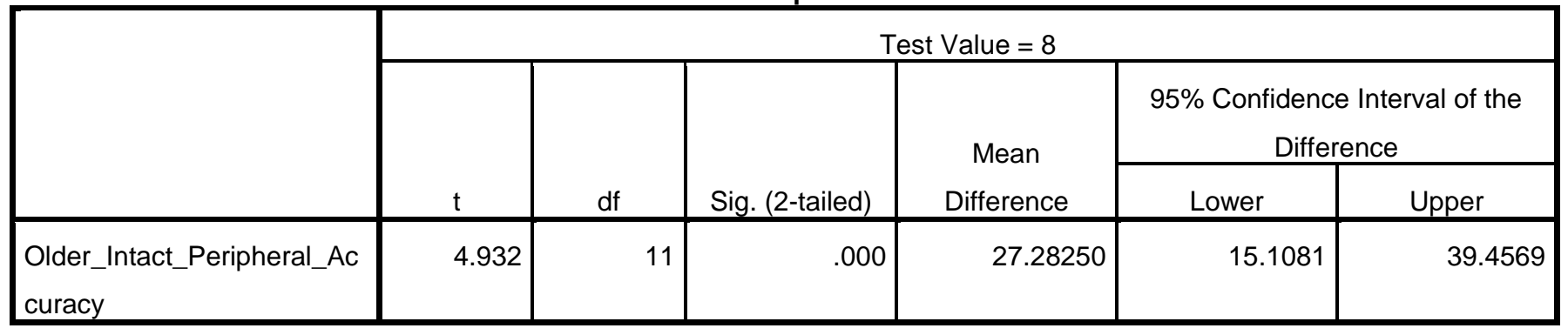

One-sample Wilcoxon signed rank test of FGMI exaggeration for central vision accuracy

for older adults with poor stereopsis

Hypothesis Test Summary

\begin{tabular}{|c|c|c|c|c|}
\hline & Null Hypothesis & Test & Sig. & Decision \\
\hline 1 & $\begin{array}{l}\text { The median of } \\
\text { Older_Poor_Peripheral_A } \\
\text { equals } 8.00 \text {. }\end{array}$ & $\begin{array}{c}\text { One-Sample } \\
\text { Accurachyilcoxon Signed } \\
\text { Rank Test }\end{array}$ & .005 & $\begin{array}{l}\text { Reject the } \\
\text { null } \\
\text { hypothesis. }\end{array}$ \\
\hline
\end{tabular}

Asymptotic significances are displayed. The significance level is .05.

Peripheral vison accuracy comparison amongst younger adults, older adults with intact stereopsis, and older adults with poor stereopsis

Test of Homogeneity of Variance

\begin{tabular}{|c|c|c|c|c|c|}
\hline & & $\begin{array}{l}\text { Levene } \\
\text { Statistic }\end{array}$ & df1 & $\mathrm{df} 2$ & Sig. \\
\hline \multirow[t]{4}{*}{ Peripheral Accuracy } & Based on Mean & 7.560 & 2 & 53 & .001 \\
\hline & Based on Median & 6.480 & 2 & 53 & .003 \\
\hline & $\begin{array}{l}\text { Based on Median and with } \\
\text { adjusted df }\end{array}$ & 6.480 & 2 & 12.799 & .011 \\
\hline & Based on trimmed mean & 6.606 & 2 & 53 & .003 \\
\hline
\end{tabular}




Report
Peripheral Accuracy
\begin{tabular}{|l|r|c|}
\hline Group & N & Median \\
\hline Younger Intact Stereo & 34 & 29.4063 \\
Older Intact Stereo & 12 & 37.0208 \\
Older Poor Stereo & 10 & 69.7143 \\
Total & 56 & 32.4583 \\
\hline
\end{tabular}

\begin{tabular}{|c|c|}
\hline \multicolumn{2}{|c|}{ Test Statistics $^{a, b}$} \\
\hline & $\begin{array}{l}\text { Peripheral } \\
\text { Accuracy }\end{array}$ \\
\hline $\begin{array}{l}\text { Chi-Square } \\
\text { df } \\
\text { Asymp. Sig. }\end{array}$ & $\begin{array}{r}4.878 \\
2 \\
.087\end{array}$ \\
\hline
\end{tabular}

a. Kruskal Wallis Test

b. Grouping Variable: Group

Peripheral vision accuracy comparison between younger adults and older adults with intact stereopsis

\begin{tabular}{|l|r|}
\hline \multicolumn{2}{|c|}{ Test Statistics $^{\mathbf{a}}$} \\
\hline & \multicolumn{1}{|c|}{ Peripheral } \\
& Accuracy \\
\hline Mann-Whitney U & 186.000 \\
Wilcoxon W & 781.000 \\
Z & -.450 \\
Asymp. Sig. (2-tailed) & .653 \\
\hline
\end{tabular}

a. Grouping Variable: Group

Peripheral vision accuracy comparison between younger adults and older adults with poor stereopsis

\begin{tabular}{|l|r|}
\multicolumn{2}{c}{ Test Statistics $^{\mathbf{b}}$} \\
\hline Mann-Whitney U & Peripheral \\
Accuracy \\
Wilcoxon W & 96.000 \\
Z & 691.000 \\
Asymp. Sig. (2-tailed) & -2.072 \\
Exact Sig. [2*(1-tailed Sig.)] & .038 \\
\hline
\end{tabular}

a. Not corrected for ties.

b. Grouping Variable: Group 


\section{Peripheral vision accuracy comparison between the two older adult groups}

\begin{tabular}{|l|r|}
\hline \multicolumn{2}{|c|}{ Test Statistics $^{\mathbf{b}}$} \\
\hline & $\begin{array}{c}\text { Peripheral } \\
\text { Accuracy }\end{array}$ \\
\hline Mann-Whitney U & 32.000 \\
Wilcoxon W & 110.000 \\
Z & -1.846 \\
Asymp. Sig. (2-tailed) & .065 \\
Exact Sig. [2*(1-tailed Sig.)] & $.069^{*}$ \\
\hline
\end{tabular}

a. Not corrected for ties.

b. Grouping Variable: Group

\section{Central vison precision comparison amongst younger adults, older adults with intact}

\section{stereopsis, and older adults with poor stereopsis}

Tests of Normality

\begin{tabular}{|ll|r|r|r|r|r|r|}
\hline & \multicolumn{2}{|c|}{ Kolmogorov-Smirnova } & \multicolumn{3}{|c|}{ Shapiro-Wilk } \\
\cline { 2 - 7 } & Group & Statistic & \multicolumn{1}{c|}{ df } & \multicolumn{1}{c|}{ Sig. } & \multicolumn{1}{c|}{ Statistic } & \multicolumn{1}{c|}{ df } & \multicolumn{1}{c|}{ Sig. } \\
\hline Central Precision & Younger Intact Stereo & .191 & 34 & .003 & .867 & 34 & .001 \\
& Older Intact Stereo & .218 & 11 & .152 & .852 & 11 & .045 \\
& Older Poor Stereo & .255 & 10 & .064 & .790 & 10 & .011 \\
\hline
\end{tabular}

a. Lilliefors Significance Correction

Test of Homogeneity of Variance

\begin{tabular}{|c|c|c|c|c|c|}
\hline & & $\begin{array}{l}\text { Levene } \\
\text { Statistic }\end{array}$ & df1 & df2 & Sig. \\
\hline \multirow[t]{4}{*}{ Central Precision } & Based on Mean & 5.174 & 2 & 52 & .009 \\
\hline & Based on Median & 2.170 & 2 & 52 & .124 \\
\hline & $\begin{array}{l}\text { Based on Median and with } \\
\text { adjusted df }\end{array}$ & 2.170 & 2 & 22.620 & .137 \\
\hline & Based on trimmed mean & 4.806 & 2 & 52 & .012 \\
\hline
\end{tabular}

Report

Central Precision

\begin{tabular}{|l|r|r|}
\hline Group & N & \multicolumn{1}{|c|}{ Median } \\
\hline Younger Intact Stereo & 34 & 8.6174 \\
Older Intact Stereo & 11 & 6.2835 \\
Older Poor Stereo & 10 & 11.9465 \\
Total & 55 & 9.9265 \\
\hline
\end{tabular}

\begin{tabular}{|c|c|}
\hline & $\begin{array}{c}\text { Central } \\
\text { Precision }\end{array}$ \\
\hline $\begin{array}{l}\text { Chi-Square } \\
\text { df } \\
\text { Asymp. Sig. }\end{array}$ & $\begin{array}{r}3.173 \\
2 \\
.205\end{array}$ \\
\hline
\end{tabular}

a. Kruskal Wallis Test

b. Grouping Variable: Group 
Peripheral vison precision comparison amongst younger adults, older adults with intact stereopsis, and older adults with poor stereopsis

Tests of Normality

\begin{tabular}{|ll|r|r|r|r|r|r|}
\hline \multirow{2}{*}{} & Group & \multicolumn{3}{|c|}{ Kolmogorov-Smirnov } & \multicolumn{3}{|c|}{ Shapiro-Wilk } \\
\cline { 2 - 7 } & Statistic & \multicolumn{1}{c|}{ df } & \multicolumn{1}{c|}{ Sig. } & Statistic & \multicolumn{1}{c|}{ df } & \multicolumn{1}{c|}{ Sig. } \\
\hline Peripheral Precision & Younger Intact Stereo & .236 & 34 & .000 & .637 & 34 & .000 \\
& Older Intact Stereo & .163 & 12 & $.200^{*}$ & .928 & 12 & .362 \\
& Older Poor Stereo & .258 & 10 & .058 & .777 & 10 & .008 \\
\hline
\end{tabular}

a. Lilliefors Significance Correction

*. This is a lower bound of the true significance.

Test of Homogeneity of Variance

\begin{tabular}{|c|c|c|c|c|c|}
\hline & & $\begin{array}{l}\text { Levene } \\
\text { Statistic }\end{array}$ & df1 & df2 & Sig. \\
\hline \multirow[t]{4}{*}{ Peripheral Precision } & Based on Mean & 4.794 & 2 & 53 & .012 \\
\hline & Based on Median & 2.344 & 2 & 53 & .106 \\
\hline & Based on Median and with & 2.344 & 2 & 33.444 & .112 \\
\hline & Based on trimmed mean & 3.781 & 2 & 53 & .029 \\
\hline
\end{tabular}

Peripheral Precision

\begin{tabular}{|l|r|r|}
\hline Group & N & \multicolumn{1}{|c|}{ Median } \\
\hline Younger Intact Stereo & 34 & 16.0696 \\
Older Intact Stereo & 12 & 12.2525 \\
Older Poor Stereo & 10 & 22.1207 \\
Total & 56 & 16.7469 \\
\hline
\end{tabular}

\begin{tabular}{|l|r|}
\hline \multicolumn{2}{|c|}{ Test Statistics ${ }^{\mathbf{a}, \mathbf{b}}$} \\
\hline & $\begin{array}{c}\text { Peripheral } \\
\text { Precision }\end{array}$ \\
\hline Chi-Square & 2.012 \\
df & 2 \\
Asymp. Sig. & .366 \\
\hline
\end{tabular}

a. Kruskal Wallis Test

b. Grouping Variable: Group 
Vision accuracy difference scores comparison amongst younger adults, older adults with intact stereopsis, and older adults with poor stereopsis

\begin{tabular}{|c|c|c|c|c|c|c|c|}
\hline & \multirow[b]{2}{*}{ Group } & \multicolumn{3}{|c|}{ Kolmogorov-Smirnova } & \multicolumn{3}{|c|}{ Shapiro-Wilk } \\
\hline & & Statistic & df & Sig. & Statistic & df & Sig. \\
\hline \multirow[t]{3}{*}{$\begin{array}{l}\text { Accuracy Difference } \\
\text { Scores }\end{array}$} & $\begin{array}{l}\text { Younger Intact } \\
\text { Stereo }\end{array}$ & .147 & 34 & .059 & .893 & 34 & .003 \\
\hline & Older Intact Stereo & .155 & 11 & $.200^{*}$ & .895 & 11 & .161 \\
\hline & Older Poor Stereo & .392 & 10 & .000 & .561 & 10 & .000 \\
\hline
\end{tabular}

a. Lilliefors Significance Correction

*. This is a lower bound of the true significance.

Test of Homogeneity of Variance

\begin{tabular}{|c|c|c|c|c|c|}
\hline & & $\begin{array}{l}\text { Levene } \\
\text { Statistic }\end{array}$ & df1 & $\mathrm{df} 2$ & Sig. \\
\hline \multirow{4}{*}{$\begin{array}{l}\text { Accuracy Difference } \\
\text { Scores }\end{array}$} & Based on Mean & 3.777 & 2 & 52 & .029 \\
\hline & Based on Median & 1.599 & 2 & 52 & .212 \\
\hline & $\begin{array}{l}\text { Based on Median and } \\
\text { with adjusted df }\end{array}$ & 1.599 & 2 & 12.779 & .240 \\
\hline & Based on trimmed mean & 2.129 & 2 & 52 & .129 \\
\hline
\end{tabular}

Accuracy Difference

\begin{tabular}{|l|r|r|}
\hline Group & $\mathrm{N}$ & \multicolumn{1}{|c|}{ Median } \\
\hline Younger Intact Stereo & 34 & 8.7813 \\
Older Intact Stereo & 11 & 15.6714 \\
Older Poor Stereo & 10 & 26.8750 \\
Total & 55 & 12.2143 \\
\hline
\end{tabular}

Test Statistics ${ }^{\mathrm{a}, \mathrm{b}}$

\begin{tabular}{|l|r|}
\hline & $\begin{array}{c}\text { Accuracy } \\
\text { Difference } \\
\text { Scores }\end{array}$ \\
\hline Chi-Square & 4.615 \\
df & 2 \\
Asymp. Sig. & .100 \\
\hline
\end{tabular}

a. Kruskal Wallis Test

b. Grouping Variable: Group 
Vision accuracy difference scores comparison between younger adults and older adults with intact stereopsis

\begin{tabular}{|l|r|}
\hline \multicolumn{1}{|c|}{ Test Statistics $^{\mathbf{b}}$} \\
\hline & $\begin{array}{c}\text { Accuracy } \\
\text { Difference } \\
\text { Scores }\end{array}$ \\
\hline Mann-Whitney U & 135.000 \\
Wilcoxon W & 730.000 \\
Z & -1.373 \\
Asymp. Sig. (2-tailed) & .170 \\
Exact Sig. [2*(1-tailed Sig.)] & $.176^{\mathrm{a}}$ \\
\hline
\end{tabular}

a. Not corrected for ties.

b. Grouping Variable: Group

Vision accuracy difference scores comparison between younger adults and older adults with poor stereopsis

\begin{tabular}{|l|r|}
\hline \multicolumn{2}{|c|}{ Test Statistics $^{\mathbf{b}}$} \\
\hline & $\begin{array}{c}\text { Accuracy } \\
\text { Difference } \\
\text { Scores }\end{array}$ \\
\hline Mann-Whitney U & 102.000 \\
Wilcoxon W & 697.000 \\
Z & -1.904 \\
Asymp. Sig. (2-tailed) & .057 \\
Exact Sig. [2*(1-tailed Sig.)] & $.058^{\mathrm{a}}$ \\
\hline
\end{tabular}

a. Not corrected for ties.

b. Grouping Variable: Group

Vision accuracy difference scores comparison between the two older adult groups

\begin{tabular}{|l|r|}
\hline \multicolumn{2}{|c|}{ Test Statistics $^{\mathbf{b}}$} \\
& $\begin{array}{c}\text { Accuracy } \\
\text { Difference } \\
\text { Scores }\end{array}$ \\
\hline Mann-Whitney U & 46.000 \\
Wilcoxon W & 112.000 \\
Z & -.634 \\
Asymp. Sig. (2-tailed) & .526 \\
Exact Sig. [2*(1-tailed Sig.)] & $.557^{\mathrm{a}}$ \\
\hline
\end{tabular}

a. Not corrected for ties.

b. Grouping Variable: Group 
Vision precision difference scores comparison between younger adults, older adults with intact stereopsis, and older adults with poor stereopsis

Tests of Normality

\begin{tabular}{|c|c|c|c|c|c|c|c|}
\hline & \multirow[b]{2}{*}{ Group } & \multicolumn{3}{|c|}{ Kolmogorov-Smirnova } & \multicolumn{3}{|c|}{ Shapiro-Wilk } \\
\hline & & Statistic & $d f$ & Sig. & Statistic & df & Sig. \\
\hline \multirow[t]{3}{*}{$\begin{array}{l}\text { Precision Difference } \\
\text { Scores }\end{array}$} & $\begin{array}{l}\text { Younger Intact } \\
\text { Stereo }\end{array}$ & .235 & 34 & .000 & .627 & 34 & .000 \\
\hline & Older Intact Stereo & .217 & 11 & .155 & .880 & 11 & .103 \\
\hline & Older Poor Stereo & .250 & 10 & .076 & .796 & 10 & .013 \\
\hline
\end{tabular}

a. Lilliefors Significance Correction

Test of Homogeneity of Variance

\begin{tabular}{|ll|r|r|r|r|}
\hline & & Levene Statistic & df1 & \multicolumn{1}{c|}{ df2 } & \multicolumn{1}{c|}{ Sig. } \\
\hline Precision Difference Scores & Based on Mean & 2.219 & 2 & 52 & .119 \\
& Based on Median & 1.208 & 2 & 52 & .307 \\
& Based on Median and with & 1.208 & 2 & 42.240 & .309 \\
& adjusted df & & & \\
& Based on trimmed mean & 1.856 & 2 & 52 & .166 \\
\hline
\end{tabular}

Report

Precision Difference Scores

\begin{tabular}{|l|r|r|}
\hline Group & N & \multicolumn{1}{|c|}{ Median } \\
\hline Younger Intact Stereo & 34 & 4.9029 \\
Older Intact Stereo & 11 & 5.2058 \\
Older Poor Stereo & 10 & 7.2755 \\
Total & 55 & 5.3337 \\
\hline
\end{tabular}

Test Statistics ${ }^{\mathrm{a}, \mathrm{b}}$

\begin{tabular}{|l|r|}
\hline & $\begin{array}{c}\text { Precision } \\
\text { Difference } \\
\text { Scores }\end{array}$ \\
\hline Chi-Square & 1.137 \\
$\mathrm{df}$ & 2 \\
Asymp. Sig. & .566 \\
\hline
\end{tabular}

a. Kruskal Wallis Test

b. Grouping Variable: Group 
Spearman's rank-order correlations of age and stereoacuity with central vison accuracy, peripheral vision accuracy, central vision precision, and peripheral vision precision

\begin{tabular}{rrcccc}
\hline & & $\begin{array}{c}\text { Central Vision } \\
\text { Accuracy }\end{array}$ & $\begin{array}{c}\text { Peripheral } \\
\text { Vision Accuracy }\end{array}$ & $\begin{array}{c}\text { Central Vision } \\
\text { Precision }\end{array}$ & $\begin{array}{c}\text { Peripheral } \\
\text { Vision Precision }\end{array}$ \\
\hline Age & & & & \\
& rho $(\boldsymbol{\rho})$ & .001 & -.131 & -.274 & -.030 \\
$\boldsymbol{p}$ & .996 & .561 & .230 & .894 \\
$\mathbf{N}$ & 21 & 22 & 21 & \\
& & & & \\
Stereoacuity & & & .335 & .403 \\
rho $(\boldsymbol{\rho})$ & .428 & $.468^{*}$ & .137 & .063 \\
$\mathbf{p}$ & .053 & 22 & 21 & 22 \\
$\mathbf{N}$ & 21 & & & \\
\hline
\end{tabular}

*. Correlation is significant at the 0.05 level (2-tailed).

**. Correlation is significant at the 0.01 level (2-tailed). 


\section{References}

Andersen, G. J. (2012). Aging and vision: changes in function and performance from optics to perception. Wiley Interdisciplinary Reviews: Cognitive Science, 3(3), 403-410. doi:10.1002/wcs. 1167

Archer, S. M. (2007). Monocular diplopia due to spherocylindrical refractive errors (an American Ophthalmological Society thesis). Transactions of the American Ophthalmological Society, 105, 252-271.

Ashida, H., Lingnau, A., Wall, M. B., \& Smith, A. T. (2007). fMRI adaptation reveals separate mechanisms for first-order and second-order motion. Journal of Neurophysiology, 97(2), 1319-1325. doi:10.1152/jn.00723.2006

Barry, S. R. (2012). Beyond the critical period. Acquiring stereopsis in adulthood. In: Jennifer K. E. Steeves; Laurence R. Harris (eds.). Plasticity in Sensory Systems. Cambridge University Press. pp. 187-188. ISBN 978-1-107-02262-1.

Biederman-Thorson, M., Thorson, J., \& Lange, G. D. (1971). Apparent movement due to closely spaced sequentially flashed dots in the human peripheral field of vision. Vision Research, 11(9), 889-903. doi:10.1016/0042-6989(71)90210-0

Bonnel, S., Mohand-Said, S., \& Sahel, J. A. (2003). The aging of the retina. Experimental Gerontology, 38(8), 825-831. doi:10.1016/S0531-5565(03)00093-7

Brewer, A. A., \& Barton, B. (2012). Effects of healthy aging on human primary visual cortex. Health, 4(1), 695-702. doi:10.4236/health.2012.429109

Brooks-Wilson, A. R. (2013). Genetics of healthy aging and longevity. Human Genetics, 132(12), 1323-1338. doi:10.1007/s00439-013-1342-z 
Cavanagh, P., \& Mather, G. (1989). Motion: The long and short of it. Spatial Vision, 4(2), 103129. doi:10.1163/156856889X00077

Chang, L. H., Yotsumoto, Y., Salat, D. H., Andersen, G. J., Watanabe, T., \& Sasaki, Y. (2015). Reduction in the retinotopic early visual cortex with normal aging and magnitude of perceptual learning. Neurobiology of Aging, 36(1), 315-322.

doi:10.1016/j.neurobiolaging.2014.08.025

Chubb, C., \& Sperling, G. (1989). Two motion perception mechanisms revealed through distance-driven reversal of apparent motion. Proceedings of the National Academy of Sciences, 86(8), 2985-2989. doi:10.1073/pnas.86.8.2985

Claeys, K. G., Lindsey, D. T., De Schutter, E., \& Orban, G. A. (2003). A higher order motion region in human inferior parietal lobule: Evidence from fMRI. Neuron, 40(3), 631-642. doi:10.1016/S0896-6273(03)00590-7

Cowey, A., \& Rolls, E. T. (1974). Human cortical magnification factor and its relation to visual acuity. Experimental Brain Research, 21(5), 447-454. doi:10.1007/BF00237163

Csete, G., Bognár, A., Csibri, P., Kaposvári, P., \& Sáry, G. (2015). Aging alters visual processing of objects and shapes in inferotemporal cortex in monkeys. Brain Research Bulletin, 110, 76-83. doi:10.1016/j.brainresbull.2014.11.005

Cummings, S. R., Nevitt, M. C., Browner, W. S., Stone, K., Fox, K. M., Ensrud, K. E., ... \& Vogt, T. M. (1995). Risk factors for hip fracture in white women. New England Journal of Medicine, 332(12), 767-774. doi:10.1056/NEJM199503233321202

Curcio, C. A., Sloan, K. R., Kalina, R. E., \& Hendrickson, A. E. (1990). Human photoreceptor topography. Journal of Comparative Neurology, 292(4), 497-523. doi:10.1002/cne.902920402 
DeAngelis, G. C., \& Uka, T. (2003). Coding of horizontal disparity and velocity by MT neurons in the alert macaque. Journal of Neurophysiology, 89(2), 1094-1111. doi:10.1152/jn.00717.2002

Deary, I. J., Corley, J., Gow, A. J., Harris, S. E., Houlihan, L. M., Marioni, R. E., ... \& Starr, J. M. (2009). Age-associated cognitive decline. British Medical Bulletin, 92(1), 135-152. doi:10.1093/bmb/ldp033

Dolinar, R. M. D., Pleta, B. A., Salmoni, A. W., \& Johnson, A. M. (2016). MoCA cutoff score in relation to the functional assessment of seniors living in a rural Canadian community. Canadian Journal of Rural Medicine, 21(4), 101-106.

Faubert, J. (2002). Visual perception and aging. Canadian Journal of Experimental Psychology, 56(3), 164-176. doi:10.1037/h0087394

Ferguson, C. J. (2009). An effect size primer: A guide for clinicians and researchers. Professional Psychology: Research and Practice, 40(5), 532-538. doi:10.1037/a0015808

Fielder, A. R., \& Moseley, M. J. (1996). Does stereopsis matter in humans? Eye, 10(2), 233-238. doi:10.1038/eye.1996.51

Fincham, E. F. (1963). Monocular diplopia. The British Journal of Ophthalmology, 47(12), 705712.

Foster, D. H., Thorson, J., McIlwain, J. T., \& Biederman-Thorson, M. (1981). The fine-grain movement illusion: A perceptual probe of neuronal connectivity in the human visual system. Vision Research, 21(7), 1123-1128. doi:10.1016/0042-6989(81)90015-8

Gao, H., \& Hollyfield, J. G. (1992). Aging of the human retina. Differential loss of neurons and retinal pigment epithelial cells. Investigative Ophthalmology \& Visual Science, 33(1), 117. 
Garrett, D. D., Kovacevic, N., McIntosh, A. R., \& Grady, C. L. (2010). Blood oxygen leveldependent signal variability is more than just noise. Journal of Neuroscience, 30(14), 4914-4921. doi:10.1523/JNEUROSCI.5166-09.2010

Garrett, D. D., Samanez-Larkin, G. R., MacDonald, S. W., Lindenberger, U., McIntosh, A. R., \& Grady, C. L. (2013). Moment-to-moment brain signal variability: A next frontier in human brain mapping? Neuroscience \& Biobehavioral Reviews, 37(4), 610-624. doi:10.1016/j.neubiorev.2013.02.015

Goldstein, E. (2014). Sensation and perception. Belmont, CA: Wadsworth, Cengage Learning.

Gregory, R. L. (1957). Increase in "neurological noise" as a factor in aging. In 4th Congress International Association of Gerontology, 1, 314-324.

Hasher, L., \& Zacks, R. T. (1988). Working memory, comprehension, and aging: A review and a new view. Psychology of Learning and Motivation, 22, 193-225. doi:10.1016/S00797421(08)60041-9

Hubel, D. H., \& Wiesel, T. N. (1970). Stereoscopic vision in macaque monkey: cells sensitive to binocular depth in area 18 of the macaque monkey cortex. Nature, 225, 41-42. doi:10.1038/225041a0

Isobe, K., \& Motokawa, K. (1955). Functional structure of the retinal fovea and Maxwell's spot. Nature, 175(4450), 306-307. doi:10.1038/175306a0

Kim, C. B., Tom, B. W., \& Spear, P. D. (1996). Effects of aging on the densities, numbers, and sizes of retinal ganglion cells in rhesus monkey. Neurobiology of Aging, 17(3), 431-438. doi:10.1016/0197-4580(96)00038-3 
Kimble, T. D., \& Williams, R. W. (2000). Structure of the cone photoreceptor mosaic in the retinal periphery of adult humans: Analysis as a function of age, sex, and hemifield. Brain Structure and Function, 201(4), 305-316. doi:10.1007/s004290050319

Kosnik, W., Fikre, J., \& Sekuler, R. (1986). Visual fixation stability in older adults. Investigative Ophthalmology \& Visual Science, 27(12), 1720-1725.

Kosnik, W., Kline, D., Fikre, J., \& Sekuler, R. (1987). Ocular fixation control as a function of age and exposure duration. Psychology and Aging, 2(3), 302. doi:10.1037/08827974.2.3.302

Leat, S. J., Chan, L. L. L., Maharaj, P. D., Hrynchak, P. K., Mittelstaedt, A., Machan, C. M., \& Irving, E. L. (2013). Binocular vision and eye movement disorders in older adults. Investigative Ophthalmology \& Visual Science, 54(5), 3798-3805. doi:10.1167/iovs.1211582

Lee, S. Y., \& Koo, N. K. (2005). Change of stereoacuity with aging in normal eyes. Korean Journal of Ophthalmology, 19(2), 136-139. doi:10.3341/kjo.2005.19.2.136

Liang, Z., Yang, Y., Li, G., Zhang, J., Wang, Y., Zhou, Y., \& Leventhal, A. G. (2010). Aging affects the direction selectivity of MT cells in rhesus monkeys. Neurobiology of Aging, 31(5), 863-873. doi:10.1016/j.neurobiolaging.2008.06.013

Lin, J., Epel, E., \& Blackburn, E. (2012). Telomeres and lifestyle factors: roles in cellular aging. Mutation Research/Fundamental and Molecular Mechanisms of Mutagenesis, 730(1), 8589. doi:10.1016/j.mrfmmm.2011.08.003

Livingstone, M., \& Hubel, D. (1988). Segregation of form, color, movement, and depth: Anatomy, physiology, and perception. Science, 240(4853), 740-749. doi: $10.1126 /$ science. 3283936 
Lombardo, M., Serrao, S., Ducoli, P., \& Lombardo, G. (2013). Eccentricity dependent changes of density, spacing and packing arrangement of parafoveal cones. Ophthalmic and Physiological Optics, 33(4), 516-526. doi:10.1111/opo.12053

López-Otín, C., Blasco, M. A., Partridge, L., Serrano, M., \& Kroemer, G. (2013). The hallmarks of aging. Cell, 153(6), 1194-1217. doi:10.1016/j.cell.2013.05.039

Lord, S. R., \& Dayhew, J. (2001). Visual risk factors for falls in older people. Journal of the American Geriatrics Society, 49(5), 508-515. doi:10.1046/j.1532-5415.2001.49107.x

Loughman, J., Davison, P. A., Nolan, J. M., Akkali, M. C., \& Beatty, S. (2010). Macular pigment and its contribution to visual performance and experience. Journal of Optometry, 3(2), 74-90. doi:10.1016/S1888-4296(10)70011-X

MacDonald, S. W., Li, S. C., \& Bäckman, L. (2009). Neural underpinnings of within-person variability in cognitive functioning. Psychology and Aging, 24(4), 792-808. doi:10.1037/a0017798

MacDonald, S. W., Nyberg, L., \& Bäckman, L. (2006). Intra-individual variability in behavior: Links to brain structure, neurotransmission and neuronal activity. Trends in Neurosciences, 29(8), 474-480. doi:10.1016/j.tins.2006.06.011

Menz, M. D., \& Freeman, R. D. (2003). Stereoscopic depth processing in the visual cortex: a coarse-to-fine mechanism. Nature Neuroscience, 6(1), 59-65. doi:10.1038/nn986

Michael, R., \& Bron, A. J. (2011). The ageing lens and cataract: A model of normal and pathological ageing. Philosophical Transactions of the Royal Society of London B: Biological Sciences, 366(1568), 1278-1292. doi:10.1098/rstb.2010.0300

Nasreddine, Z. S., Phillips, N. A., Bédirian, V., Charbonneau, S., Whitehead, V., Collin, I., ... \& Chertkow, H. (2005). The Montreal Cognitive Assessment, MoCA: a brief screening tool 
for mild cognitive impairment. Journal of the American Geriatrics Society, 53(4), 695 699. doi:10.1111/j.1532-5415.2005.53221.x

Neri, P., Bridge, H., \& Heeger, D. J. (2004). Stereoscopic processing of absolute and relative disparity in human visual cortex. Journal of Neurophysiology, 92(3), 1880-1891. doi:10.1152/jn.01042.2003

Nevitt, M. C., Cummings, S. R., Kidd, S., \& Black, D. (1989). Risk factors for recurrent nonsyncopal falls: a prospective study. JAMA, 261(18), 2663-2668. doi:10.1001/jama.1989.03420180087036

Niccoli, T., \& Partridge, L. (2012). Ageing as a risk factor for disease. Current Biology, 22(17), R741-R752. doi:10.1016/j.cub.2012.07.024

Norman, J. F., Norman, H. F., Craft, A. E., Walton, C. L., Bartholomew, A. N., Burton, C. L., ... \& Crabtree, C. E. (2008). Stereopsis and aging. Vision Research, 48(23), 2456-2465. doi:10.1016/j.visres.2008.08.008

Owsley, C. (2011). Aging and vision. Vision Research, 51(13), 1610-1622. doi:10.1016/j.visres.2010.10.020

Peters, A., Moss, M. B., \& Sethares, C. (2000). Effects of aging on myelinated nerve fibers in monkey primary visual cortex. Journal of Comparative Neurology, 419(3), 364-376. doi:10.1002/(SICI)1096-9861(20000410)419:3<364::AID-CNE8>3.0.CO;2-R

Peters, A., Sethares, C., \& Killiany, R. J. (2001). Effects of age on the thickness of myelin sheaths in monkey primary visual cortex. Journal of Comparative Neurology, 435(2), 241-248. doi:10.1002/cne.1205

Precision Vision. (2017). Stereo Fly Test. Retrieved from www.precisionvision.com/product/stereo-fly-test/ 
Reed, M. J., \& Burdett, F. (2002). Apparent motion processing in strabismic observers with varying levels of stereo vision. Behavioural Brain Research, 133(2), 383-390. doi:10.1016/S0166-4328(02)00037-2

Rosenthal, R. (1991). Meta-analytic procedures for social research (Rev. ed.). Newbury Park, CA: Sage.

Rossetti, H. C., Lacritz, L. H., Cullum, C. M., \& Weiner, M. F. (2011). Normative data for the Montreal Cognitive Assessment (MoCA) in a population-based sample. Neurology, 77(13), 1272-1275. doi:10.1212/WNL.0b013e318230208a

Rubin, G. S., Ng, E. S., Bandeen-Roche, K., Keyl, P. M., Freeman, E. E., \& West, S. K. (2007). A prospective, population-based study of the role of visual impairment in motor vehicle crashes among older drivers: the SEE study. Investigative Ophthalmology and Visual Science, 48(4), 1483. doi:10.1167/iovs.06-0474

Salthouse, T. A., \& Lichty, W. (1985). Tests of the neural noise hypothesis of age-related cognitive change. Journal of Gerontology, 40(4), 443-450. doi:10.1093/geronj/40.4.443

Salvi, S. M., Akhtar, S., \& Currie, Z. (2006). Ageing changes in the eye. Postgraduate Medical Journal, 82(971), 581-587. doi:10.1136/pgmj.2005.040857

Sekuler, A. B., Bennett, J. P., \& Mamelak, M. (2000). Effects of aging on the useful field of view. Experimental Aging Research, 26(2), 103-120. doi:10.1080/036107300243588

Spear, P. D. (1993). Neural bases of visual deficits during aging. Vision Research, 33(18), 25892609. doi:10.1016/0042-6989(93)90218-L

Strasburger, H., Rentschler, I., \& Jüttner, M. (2011). Peripheral vision and pattern recognition: A review. Journal of Vision, 11(5):13, 1-82 doi:10.1167/11.5.13 
Tarita-Nistor, L., Brent, M. H., Steinbach, M. J., \& González, E. G. (2012). Fixation patterns in maculopathy: From binocular to monocular viewing. Optometry and Vision Science, 89(3), 277-287. doi:10.1097/OPX.0b013e318244e8b1

Thompson, A. M., \& Nawrot, M. (1999). Abnormal depth perception from motion parallax in amblyopic observers. Vision Research, 39(7), 1407-1413. doi:10.1016/S00426989(98)00235-1

Vaina, L. M., \& Soloviev, S. (2004). First-order and second-order motion: Neurological evidence for neuroanatomically distinct systems. Progress in Brain Research, 144, 197 212. doi:10.1016/S0079-6123(03)14414-7

Voytek, B., Kramer, M. A., Case, J., Lepage, K. Q., Tempesta, Z. R., Knight, R. T., \& Gazzaley, A. (2015). Age-related changes in 1/f neural electrophysiological noise. Journal of Neuroscience, 35(38), 13257-13265. doi:10.1523/JNEUROSCI.2332-14.2015

Wheatstone, C. (1838). Contributions to the physiology of vision.-Part the first. On some remarkable, and hitherto unobserved, phenomena of binocular vision. Philosophical Transactions of the Royal Society of London, 128(1838), 371-394. Retrieved from www.jstor.org/stable/108203

Woods, R. L., Bradley, A., \& Atchison, D. A. (1996). Monocular diplopia caused by ocular aberrations and hyperopic defocus. Vision Research, 36(22), 3597-3606. doi:10.1016/0042-6989(96)00092-2

Wright, L. A., \& Wormald, R. P. L. (1992). Stereopsis and ageing. Eye, 6(5), 473-476. doi:10.1038/eye.1992.100 
Yang, Y., Liang, Z., Li, G., Wang, Y., \& Zhou, Y. (2009). Aging affects response variability of V1 and MT neurons in rhesus monkeys. Brain Research, 1274, 21-27. doi:10.1016/j.brainres.2009.04.015

Yang, Y., Liang, Z., Li, G., Wang, Y., Zhou, Y., \& Leventhal, A. G. (2008). Aging affects contrast response functions and adaptation of middle temporal visual area neurons in rhesus monkeys. Neuroscience, 156(3), 748-757. doi:10.1016/j.neuroscience.2008.08.007

Yang, Y., Zhang, J., Liang, Z., Li, G., Wang, Y., Ma, Y., ... \& Leventhal, A. G. (2009). Aging affects the neural representation of speed in macaque area MT. Cerebral Cortex, 19(9), 1957-1967. doi:10.1093/cercor/bhn221

Yeo, B. T., Krienen, F. M., Sepulcre, J., Sabuncu, M. R., Lashkari, D., Hollinshead, M., ... \& Fischl, B. (2011). The organization of the human cerebral cortex estimated by intrinsic functional connectivity. Journal of Neurophysiology, 106(3), 1125-1165.

doi:10.1152/jn.00338.2011 


\section{Glossary}

Age-related macular degeneration: Damage that occurs to the macula in the context of aging, resulting in loss of central vision; dry age-related macular degeneration, the more common form, is due to macula thinning and the growth of clumps of protein; wet age-related macular degeneration is due to scarring of the macula caused by the leaking of abnormal blood vessels growing under the retina

Area middle temporal (MT)/V5: region of extrastriate visual cortex believed to be integral to motion perception due to its high density of direction sensitive neurons

Astigmatism: a refractive error in which light is not focused evenly on the retina

Beta movement: The perceptual illusion of an object moving when two or more static images are presented in succession

Binocular disparity: the difference in an object's location between the left and right eye images Central vision: The sharpest form of vision which, in individuals with normal fixation, results from the eye fixating on an target so that light reflected from the target falls within the fovea; the central $1.5-2$ degrees of the visual field

Cones: retinal photoreceptors responsible for colour vision

Cornea: the transparent front layer of the eye

Corollary discharge: a copy of the motor command to the eye, which is sent to other regions of the brain to inform it of the eye's movement

Corrected visual acuity: a participant's visual acuity when wearing their corrective lenses

Diabetic retinopathy: An eye condition associated with diabetes in which blood vessels of the retina leak or bleed, resulting in distorted vision 
Esophoria: deviation of the eye inward when binocular fusion of the left and right eye images does not occur

Exophoria: deviation of the eye outward when binocular fusion of the left and right eye images does not occur

Fixation: The stable focus of the eye(s) on a point of the visual field, typically to bring a target into focus or to track its position

Fovea: The area of the macula responsible for sharp central vision

Foveola: The centre of the fovea

Foveal vision: See central vision

Ganglion layer: layer of cells that receives visual information from photoreceptors

Glaucoma: A group of diseases that damage the optic nerve, potentially resulting in vision loss or blindness

Lateral geniculate nucleus: area within the thalamus that relays visual information to the primary visual cortex

Lens: The transparent structure that helps focus light on the retina

Macula: The yellow oval spot at the centre of the retina that contains the fovea Magnocellular pathway: the visual pathway dealing with motion and spatial analysis

Myelin: A fatty sheath-like material that insulates the axon of some nerve cells Nasal mid-periphery: The part of the visual field $30-60^{\circ}$ towards the nose Optic nerve: the nerve that carries visual information from the retina to the brain Peripheral vision: The form of vision which, in individuals with normal fixation, results when light from the target falls outside the fovea; any part of the visual field outside of central vision 
Phoria: deviation of the eyes when binocular fusion of the left and right eye images does not occur

Photoreceptor: a rod or cone

Primary visual cortex: The area of the visual cortex that receives visual information from the lateral geniculate nucleus

Receptive field: the region of sensory space to which a specific neuron will respond

Retina: the light-sensitive layer of tissue at the back of the eye

Retinal pigment epithelial cells: pigmented cells that nourish retinal visual cells

Rods: retinal photoreceptors that can function in less intense light than cones due to greater sensitivity

Stereoacuity: The smallest difference in the images presented to the right and left eye that can be detected reliably as binocular depth

Stereopsis: The binocular perception of depth produced by the brain's interpretation of the different images presented to the right and left eyes

Strabismus: A condition characterized by inward or outward misalignment of the eye Temporal mid-periphery: The part of the visual field $30-60^{\circ}$ towards the temples Thalamus: brain region involved in relaying sensory information from receptors to cerebral cortex

V1: See primary visual cortex

V2, V3: Extrastriate regions of the visual cortex

Visual angle: The angle formed at the eye by the light rays reflected from an object 